\title{
THE EXISTENCE AND CONSTRUCTION OF RATIONAL GAUSS-TYPE QUADRATURE RULES*
}

\author{
KARL DECKERS ${ }^{\dagger}$ AND ADHEMAR BULTHEEL ${ }^{\ddagger}$
}

\begin{abstract}
Consider a hermitian positive-definite linear functional $\mathfrak{F}$, and assume we have $m$ distinct nodes fixed in advance anywhere on the real line. In this paper we then study the existence and construction of $n$th rational Gauss-Radau $(m=1)$ and Gauss-Lobatto $(m=2)$ quadrature formulas that approximate $\mathfrak{F}\{f\}$. These are quadrature formulas with $n$ positive weights and with the $n-m$ remaining nodes real and distinct, so that the quadrature is exact in a $(2 n-m)$-dimensional space of rational functions.
\end{abstract}

Key words. Quasi-orthogonal rational functions, generalized eigenvalue problem, positive rational interpolatory quadrature rules.

AMS subject classifications. 42C05, 65D32, 65F 15

1. Introduction. The central object of study in this paper is the numerical approximation of a hermitian positive-definite functional $\mathfrak{F}$ by means of an $n$-point positive interpolatory quadrature rule with real distinct nodes $\left\{x_{k}\right\}_{k=1}^{n}$ and positive weights $\left\{\lambda_{k}\right\}_{k=1}^{n}$ :

$$
\mathfrak{F}\{f\} \approx \sum_{k=1}^{n} \lambda_{k} f\left(x_{k}\right) .
$$

When taking as nodes the zeros of an $n$th orthogonal polynomial (OP) (where orthogonality is with respect to the inner product defined by $\langle f, g\rangle=\mathfrak{F}\{f(x) \cdot \overline{g(\bar{x})}\})$, we obtain the $n$th Gaussian quadrature rule, which has a maximal polynomial domain of validity; i.e., the approximation is exact for every polynomial of degree less than or equal to $2 n-1$. Clearly, the existence of the $n$th Gaussian quadrature rule depends on whether an $n$th OP exists. If one or two nodes in the quadrature formula are fixed in advance, and the remaining nodes are chosen to obtain exactness for at least every polynomial of degree less than or equal to $2 n-2$ or $2 n-3$ respectively, we get more general $n$th Gauss-type quadrature rules. In this case, the nodes are zeros of an $n$th quasi-orthogonal polynomial, and the existence depends on the fixed node(s) as well (i.e., for certain choices, one of the remaining nodes may lie at infinity and/or some of the corresponding weights may be non-positive).

When $f$ has singularities, it is often more appropriate to not consider a maximal polynomial domain of validity, but rather consider more general spaces of rational functions. In such a case the OPs are replaced by orthogonal rational functions (ORFs) with preassigned poles (to simulate the singularities of $f$ ).

ORFs were first introduced by Džrbašian in the 1960s. Most of his papers appeared in Russian literature, but an accessible survey in English can be found in $[9,13]$. These ORFs are a generalization of OPs in such a way that they are of increasing degree with a given sequence of poles, and the OPs result if all the poles are at infinity.

${ }^{*}$ This work is partially supported by the Belgian Network DYSCO (Dynamical Systems, Control, and Optimization), funded by the Interuniversity Attraction Poles Programme, initiated by the Belgian State, Science Policy Office. The scientific responsibility rests with its authors.

$\dagger$ Department of Computer Science, Katholieke Universiteit Leuven, Heverlee, Belgium. This author is a Postdoctoral Fellow of the Research Foundation - Flanders (FWO).

${ }^{\ddagger}$ Department of Computer Science, Katholieke Universiteit Leuven, Heverlee, Belgium. 
So, when taking as nodes the zeros of an $n$th ORF instead, we obtain the $n$th rational Gaussian quadrature rule; see e.g. [1, Chapt. 11.6] and $[2,7,8,10,11,12$, $14,15,17,18,19,20]$. Here we can also consider more general rational Gauss-type quadrature rules obtained by fixing one or two nodes in the quadrature rule. However, the existence of these quadrature rules now also depends on the sequence of poles.

The aim of this paper is to investigate the existence and construction of rational Gauss-type quadrature rules. The outline is as follows. After giving the necessary theoretical background in Section 2, in Section 3 we discuss the connection of positive rational interpolatory quadrature formulas with certain kinds of quasi-orthogonal rational functions. Next, in Section 4 we use this connection to study the existence and construction of rational Gauss-type quadrature formulas for a given (set of) node(s) fixed in advance. Finally, some illustrative numerical examples are given in Section 5.

2. Preliminaries. The field of complex numbers will be denoted by $\mathbb{C}$ and the Riemann sphere by $\overline{\mathbb{C}}=\mathbb{C} \cup\{\infty\}$. For the real line we use the symbol $\mathbb{R}$ and for the extended real line $\overline{\mathbb{R}}=\mathbb{R} \cup\{\infty\}$. Further, the positive half line will be represented by $\mathbb{R}^{+}=\{x \in \mathbb{R}: x \geq 0\}$. Whenever the value zero is omitted in a set $X \subseteq \overline{\mathbb{C}}$, this will be represented by $X_{0}$. Similarly, the complement of a set $Y \subset \overline{\mathbb{C}}$ with respect to a set $X \subseteq \overline{\mathbb{C}}$ will be given by $X_{Y}$; i.e., $X_{Y}=\{t \in X: t \notin Y\}$.

Let $a \in \mathbb{C}$, then $\mathcal{R} e\{a\}$ refers to the real part of $a$, while $\operatorname{Im}\{a\}$ refers to the imaginary part, and the imaginary unit will be denoted by i. For $a \neq 0$ we will also use the short notation $\mathfrak{T}\{a\}$, defined by

$$
\mathfrak{T}\{a\}=-\mathcal{I} m\{1 / a\}= \begin{cases}\frac{\mathcal{I} m\{a\}}{|a|^{2}}, & a \in \mathbb{C}_{0} \\ 0, & a=\infty\end{cases}
$$

For any complex function $f$, we define the involution operation or super-c conjugate by $f^{c}(x)=\overline{f(\bar{x})}$. With $\mathcal{P}_{k}$ we denote the space of polynomials of degree less than or equal to $k$, while $\mathcal{P}$ represents the space of all polynomials. Further, we will use the short notation $[f(x)]_{x=a}$ to denote $\lim _{x \rightarrow a} f(x)$.

Let a sequence of poles $\mathcal{A}=\left\{\alpha_{1}, \alpha_{2}, \ldots\right\} \subset \overline{\mathbb{C}}_{0}$ be fixed, where the poles are arbitrary complex or infinite; hence, they do not have to appear in pairs of complex conjugates. The rational functions we then deal with, are of the form

$$
f_{k}(x)=\frac{c_{k} x^{k}+c_{k-1} x^{k-1}+\cdots+c_{0} x^{0}}{\left(1-x / \alpha_{1}\right)\left(1-x / \alpha_{2}\right) \cdots\left(1-x / \alpha_{k}\right)}, \quad k=1,2, \ldots
$$

Note that, whenever $\alpha_{j}=\infty$ for every $j \geqslant 1$, the "rational functions" $f_{k}(x)$ in $(2.1)$ are in fact polynomials of degree $k$. Thus the polynomial case is automatically accounted for.

Define the factors

$$
Z_{k}(x):=Z_{\alpha_{k}}(x)=\frac{x}{1-x / \alpha_{k}}, \quad k=1,2, \ldots,
$$

and the basis functions

$$
b_{0}(x) \equiv 1, \quad b_{k}(x)=b_{k-1}(x) Z_{k}(x), \quad k=1,2, \ldots .
$$

These basis functions generate the nested spaces of rational functions with poles in $\mathcal{A}$ defined by

$$
\mathcal{L}_{-1}=\{0\}, \quad \mathcal{L}_{0}=\mathbb{C}, \quad \mathcal{L}_{k}:=\mathcal{L}\left\{\alpha_{1}, \ldots, \alpha_{k}\right\}=\operatorname{span}\left\{b_{0}, \ldots, b_{k}\right\}, k=1,2, \ldots
$$


With $\mathcal{L}$ we then denote the closed linear span of all $\left\{b_{k}\right\}_{k=0}^{\infty}$. Let

$$
\pi_{0}(x) \equiv 1, \quad \pi_{k}(x)=\prod_{j=1}^{k}\left(1-x / \alpha_{j}\right), \quad k=1,2, \ldots,
$$

then for $k \geqslant 0$ we may write equivalently

$$
b_{k}(x)=\frac{x^{k}}{\pi_{k}(x)} \quad \text { and } \quad \mathcal{L}_{k}=\left\{p_{k} / \pi_{k}: p_{k} \in \mathcal{P}_{k}\right\}
$$

In the remainder we will also use the short notation $\mathcal{L}_{k}^{[\alpha]}:=\mathcal{L}\left\{\alpha_{1}, \ldots, \alpha_{k-1}, \alpha\right\}$ (i.e., the space of rational functions with the same dimension as $\mathcal{L}_{k}$, but with the last pole $\alpha_{k}$ replaced by the pole $\alpha$ ) and $\mathcal{L}_{k}(\alpha):=\left\{f=\frac{p_{k}}{\pi_{k}} \in \mathcal{L}_{k}: p_{k}(\alpha)=0\right\}=\frac{Z_{k}}{Z_{\alpha}} \mathcal{L}_{k-1}$.

Note that $\mathcal{L}_{k}$ and $\mathcal{L}$ are rational generalizations of $\mathcal{P}_{k}$ and $\mathcal{P}$. Indeed, if $\alpha_{j}=\infty$ for every $j \geqslant 1$, the expression in (2.2) becomes $Z_{k}(x)=x$ and the expression in $(2.3)$ becomes $b_{k}(x)=x^{k}$.

With the definition of the super-c conjugate we introduce $\mathcal{L}_{k}^{c}=\left\{f^{c}: f \in \mathcal{L}_{k}\right\}$. Further, with $\mathcal{R}_{k, j}$ and $\mathcal{S}_{k}=\mathcal{S}\left\{\alpha_{1}, \ldots, \alpha_{k}\right\}$ we denote the space of rational functions defined by $\mathcal{R}_{k, j}=\mathcal{L}_{k} \cdot \mathcal{L}_{j}^{c}:=\left\{f \cdot g^{c}: f \in \mathcal{L}_{k}\right.$ and $\left.g \in \mathcal{L}_{j}\right\}$ and $\mathcal{S}_{k}=\mathcal{L}_{k}+\mathcal{L}_{k}^{c}:=$ $\left\{f+g^{c}: f \in \mathcal{L}_{k}\right.$ and $\left.g \in \mathcal{L}_{k}\right\}$ respectively.

Consider an inner product defined by a linear functional $\mathfrak{F}$ :

$$
\langle f, g\rangle_{\mathfrak{F}}=\mathfrak{F}\left\{f g^{c}\right\}, \quad f, g \in \mathcal{L},
$$

where the linear functional $\mathfrak{F}$ is assumed to be hermitian positive-definite (HPD); i.e.,

$$
\mathfrak{F}\left\{f g^{c}\right\}=\overline{\mathfrak{F}\left\{f^{c} g\right\}} \quad \text { and } \quad \mathfrak{F}\left\{f f^{c}\right\}>0 \text { for } f \neq 0 .
$$

Orthogonalizing the basis functions $\left\{b_{0}, b_{1}, \ldots\right\}$ with respect to this inner product, we obtain a sequence of orthogonal rational functions (ORFs) $\left\{\phi_{0}, \phi_{1}, \ldots\right\}$, with $\phi_{k} \in$ $\mathcal{L}_{k} \backslash \mathcal{L}_{k-1}$, so that $\phi_{k} \perp_{\mathfrak{F}} \mathcal{L}_{k-1}$; i.e.,

$$
\left\langle\phi_{k}, \phi_{j}\right\rangle_{\mathfrak{F}}=\frac{1}{\left|d_{k}\right|^{2}} \delta_{k, j}, \quad d_{k} \in \mathbb{C}_{0}, \quad k, j=0,1, \ldots
$$

where $\delta_{k, j}$ is the Kronecker Delta. In the special case in which $\varphi_{k}(x)=d_{k} \phi_{k}(x)$, so that

$$
\left\|\varphi_{k}\right\|_{\mathfrak{F}}:=\sqrt{\left\langle\varphi_{k}, \varphi_{k}\right\rangle_{\mathfrak{F}}}=1
$$

we say that $\varphi_{k}$ is an orthonormal rational function (nORF).

Put by convention $\alpha_{-1}=\alpha_{0}=\infty$. We then call a rational function $f_{k}=$ $\frac{p_{k}}{\pi_{k}} \in \mathcal{L}_{k} \backslash \mathcal{L}_{k-1}$, with $k \geqslant 0$, exceptional (respectively degenerate) iff $p_{k}\left(\alpha_{k-1}\right)=0$ (respectively $p_{k}\left(\bar{\alpha}_{k-1}\right)=0$ ). A zero of $p_{k}$ at $\infty$ means that the degree of $p_{k}$ is less than $k$. Further, for $k>1$ we say that $f_{k}$ is singular iff $p_{k}\left(\alpha_{j}\right)=0$ for at least one $j \in\{0, \ldots, k-2\}$. Finally, $f_{k}$ is called regular iff $f_{k}$ is not singular, not degenerate and not exceptional. With these definitions we now introduce the following spaces of rational functions:

$$
\begin{aligned}
{ }^{e} \mathcal{L}_{k} & :=\left\{f \in \mathcal{L}_{k} \backslash \mathcal{L}_{k-1}: f \text { is not exceptional }\right\}, \\
{ }^{d} \mathcal{L}_{k} & :=\left\{f \in \mathcal{L}_{k} \backslash \mathcal{L}_{k-1}: f \text { is not degenerate }\right\}, \\
{ }^{d} \mathcal{L}_{k} & :={ }^{e} \mathcal{L}_{k} \cap{ }^{d} \mathcal{L}_{k}, \\
{ }^{r} \mathcal{L}_{k} & :=\left\{f \in \mathcal{L}_{k} \backslash \mathcal{L}_{k-1}: f \text { is regular }\right\} .
\end{aligned}
$$


In [18, Thm. 2.1.1] (and [1, Chapt. 11.1] for the special case of all real poles) the following three-term recurrence relation has been proved for nORFs $\varphi_{k} \in \mathcal{L}_{k} \backslash \mathcal{L}_{k-1}$.

TheOREM 2.1. Consider the nORFs $\varphi_{j} \in \mathcal{L}_{j} \backslash \mathcal{L}_{j-1}$, with $j=k, k-1, k-2$ and $k>0$. Then these $n$ ORFs satisfy a three-term recurrence relation of the form

$$
\begin{aligned}
\varphi_{k}(x) & =E_{k} Z_{k}(x)\left\{\left[1+\frac{D_{k}}{Z_{k-1}(x)}\right] \varphi_{k-1}(x)-\frac{C_{k}}{Z_{k-2}^{c}(x)} \varphi_{k-2}(x)\right\} \\
& =E_{k} \psi_{k}(x),
\end{aligned}
$$

with $\left|E_{k}\right|=\left\|\psi_{k}\right\|_{\mathfrak{F}}^{-1} \in \mathbb{R}_{0}^{+}, C_{k} \in \mathbb{C}_{0}$ and $D_{k} \in \mathbb{C}$ iff $\varphi_{k} \in{ }^{e} \mathcal{L}_{k}$ and $\varphi_{k-1} \in{ }^{d} \mathcal{L}_{k-1}$. The initial conditions are $\alpha_{-1}=\alpha_{0}=\infty, \varphi_{-1}(x) \equiv 0$, and $\left|\varphi_{0}(x)\right| \equiv\|1\|_{\mathfrak{F}}^{-1}=\left|E_{0}\right|$. In the special case in which $\varphi_{k-1} \in{ }^{d e} \mathcal{L}_{k-1}$ and $\varphi_{k-2} \in{ }^{d} \mathcal{L}_{k-2}$ for $k>1$, it holds that (see [4, Thm. 3.3])

$$
C_{k}=\frac{1-D_{k} / Z_{k-1}^{c}\left(\alpha_{k-1}\right)}{\bar{E}_{k-1}} \in \mathbb{C}_{0}
$$

In this case the coefficient $D_{k}$ can be expressed in terms of inner products as follows (see [4, Thm. 3.7 and Thm. 3.9]):

$$
D_{k}=\frac{K_{k, j}-L_{k, j}}{\frac{L_{k, j}}{Z_{k-1}\left(\alpha_{k}\right)}+\frac{K_{k, j}}{Z_{k-1}^{c}\left(\alpha_{k-1}\right)}+\delta_{k-1, j} \bar{E}_{k-1}}, \quad 0 \leqslant j<k
$$

with

$$
K_{k, j}=\frac{1}{Z_{k-2}^{c}\left(\alpha_{k}\right)}\left\langle Z_{k} \varphi_{k-2}, \varphi_{j}\right\rangle_{\mathfrak{F}}+\delta_{k-2, j} \quad \text { and } \quad L_{k, j}=\bar{E}_{k-1}\left\langle Z_{k} \varphi_{k-1}, \varphi_{j}\right\rangle_{\mathfrak{F}},
$$

while for $\alpha_{k} \notin \overline{\mathbb{R}}$, the modulus of the coefficient $E_{k}$ can also be computed as follows:

$$
\left|E_{k}\right|^{2}=\frac{\mathfrak{T}\left\{\alpha_{k}\right\}\left|E_{k-1}\right|^{2}}{\left(\mathcal{I} m\left\{D_{k}\right\}-\left|D_{k}\right|^{2} \mathfrak{T}\left\{\alpha_{k-1}\right\}\right) \cdot\left(\left|E_{k-1}\right|^{2}-4 \mathfrak{T}\left\{\alpha_{k-1}\right\} \cdot \mathfrak{T}\left\{\alpha_{k-2}\right\}\right)+\mathfrak{T}\left\{\alpha_{k-2}\right\}} .
$$

In the opposite direction as in Theorem 2.1, the following Favard-type theorem has been proved in [3].

THEOREM 2.2. Let $\left\{\chi_{k}\right\}_{k=0}^{\infty}$ be a sequence of rational functions in $\mathcal{L}$, and assume that the following conditions are satisfied:

(A1) $\alpha_{-1}=\alpha_{0}=\infty$ and $\alpha_{k} \in \overline{\mathbb{C}}_{0}, k=1,2, \ldots$;

(A2) $\chi_{k}$ is generated by the three-term recurrence relation (2.4);

(A3) $\chi_{k} \in \mathcal{L}_{k} \backslash \mathcal{L}_{k-1}, k=0,1,2, \ldots$, and $\chi_{-1} \equiv 0$;

(A4) $D_{k} \in \mathbb{C}$ and $E_{k} \in \mathbb{C}_{0}, k=1,2, \ldots$;

(A5)

$$
\operatorname{Im}\left\{D_{k}\right\}=\frac{\mathfrak{T}\left\{\alpha_{k}\right\}}{\left|E_{k}\right|^{2}}-\frac{\mathfrak{T}\left\{\alpha_{k-2}\right\}}{\left|E_{k-1}\right|^{2}}
$$

if $\alpha_{k-1} \in \overline{\mathbb{R}}_{0}$, respectively

$$
\mathcal{R} e\left\{D_{k}\right\}^{2}+\left\{\mathcal{I} m\left\{D_{k}\right\}-\mathbf{i} Z_{k-1}\left(\bar{\alpha}_{k-1}\right)\right\}^{2}=-\left\{Z_{k-1}\left(\bar{\alpha}_{k-1}\right)\right\}^{2} \frac{\left|E_{k-1}\right|^{2}}{\left|E_{k}\right|^{2}} \cdot \frac{\Delta_{k}}{\Delta_{k-1}}
$$

if $\alpha_{k-1} \notin \overline{\mathbb{R}}$, where $\Delta_{k}=\left|E_{k}\right|^{2}-4 \mathfrak{T}\left\{\alpha_{k}\right\} \cdot \mathfrak{T}\left\{\alpha_{k-1}\right\}>0, k=1,2, \ldots$, with $E_{0} \in \mathbb{C}_{0}$ 
(A6) $C_{k}=\frac{1-D_{k} / Z_{k-1}^{c}\left(\alpha_{k-1}\right)}{\bar{E}_{k-1}} \in \mathbb{C}_{0}, k=1,2, \ldots$

Then there exists a HPD linear functional $\mathfrak{G}$ on $\mathcal{L} \cdot \mathcal{L}^{c}$ so that

$$
\langle f, g\rangle_{\mathfrak{G}}=\mathfrak{G}\left\{f g^{c}\right\}
$$

defines an inner product on $\mathcal{L}$ for which the $\chi_{k}$ form an orthonormal system.

The above three-term recurrence relation can also be written as follows:

$$
\begin{aligned}
x\left\{\varphi_{k-1}(x)-\right. & \left.\frac{a_{k-1}^{[\varphi]}}{Z_{k-2}^{c}\left(\alpha_{k-2}\right)} \varphi_{k-2}(x)\right\}=a_{k-1}^{[\varphi]}\left(1-\frac{x}{\alpha_{k-2}}\right) \varphi_{k-2}(x) \\
& +b_{k-1}^{[\varphi]}\left(1-\frac{x}{\alpha_{k-1}}\right) \varphi_{k-1}(x)+c_{k-1}^{[\varphi]}\left(1-\frac{x}{\alpha_{k}}\right) \varphi_{k}(x), \quad 0<k \leqslant n,
\end{aligned}
$$

where

$$
b_{k-1}^{[\varphi]}=-D_{k}, \quad c_{k-1}^{[\varphi]}=E_{k}^{-1}, \quad \text { and } a_{k-1}^{[\varphi]}=C_{k}=\left\{1+\frac{b_{k-1}^{[\varphi]}}{Z_{k-1}^{c}\left(\alpha_{k-1}\right)}\right\} \bar{c}_{k-2}^{[\varphi]} .
$$

Let $\mathbf{I}_{n}$ denote the $n$ by $n$ identity matrix, and define the matrices

$$
\begin{aligned}
\mathbf{J}_{n} & =\left(\begin{array}{ccccc}
b_{0}^{[\varphi]} & c_{0}^{[\varphi]} & 0 & \ldots & 0 \\
a_{1}^{[\varphi]} & b_{1}^{[\varphi]} & c_{1}^{[\varphi]} & \ddots & \vdots \\
0 & \ddots & \ddots & \ddots & 0 \\
\vdots & \ddots & a_{n-2}^{[\varphi]} & b_{n-2}^{[\varphi]} & c_{n-2}^{[\varphi]} \\
0 & \cdots & 0 & a_{n-1}^{[\varphi]} & b_{n-1}^{[\varphi]}
\end{array}\right), \mathbf{D}_{n}=\operatorname{diag}\left(\alpha_{0}^{-1}, \alpha_{1}^{-1}, \ldots, \alpha_{n-1}^{-1}\right), \\
\mathbf{S}_{n} & =\left(\begin{array}{ccccc}
0 & \ldots & \ldots & \ldots & 0 \\
\frac{a_{1}^{[\varphi]}}{Z_{0}^{c}\left(\alpha_{0}\right)} & \ddots & & & \vdots \\
0 & \ddots & \ddots & & \vdots \\
\vdots & \ddots & \ddots & \ddots & \vdots \\
0 & \ldots & 0 & \frac{a_{n-1}^{[\varphi]}}{Z_{n-2}^{c}\left(\alpha_{n-2}\right)} & 0
\end{array}\right), \text { and } \mathbf{B}_{n}=\mathbf{J}_{n} \mathbf{D}_{n}+\mathbf{I}_{n}-\mathbf{S}_{n},
\end{aligned}
$$

and column vectors

$$
\vec{\varphi}_{n}(x)=\left(\begin{array}{llll}
\varphi_{0}(x) & \varphi_{1}(x) & \ldots & \varphi_{n-1}(x)
\end{array}\right)^{T} \quad \text { and } \quad \vec{e}_{n}=\left(\begin{array}{llll}
0 & \ldots & 0 & 1
\end{array}\right)^{T} \in \mathbb{C}^{n} .
$$

Assuming the nORFs $\varphi_{k} \in{ }^{d e} \mathcal{L}_{k}$ for $k=0, \ldots, n-1$, and $\varphi_{n} \in{ }^{e} \mathcal{L}_{n}$, we obtain that

$$
\mathbf{J}_{n} \vec{\varphi}_{n}(x)=x \mathbf{B}_{n} \vec{\varphi}_{n}(x)-c_{n-1}^{[\varphi]}\left(1-\frac{x}{\alpha_{n}}\right) \varphi_{n}(x) \vec{e}_{n} .
$$

The following theorem has then be proved in [16, Sec. 4].

TheOREM 2.3. Suppose the $n$ ORFs $\varphi_{k} \in{ }^{d e} \mathcal{L}_{k}$ for $k=0, \ldots, n-1$. Then the zeros $x_{n, j}, j=1, \ldots, n$, of a $n$ ORF $\varphi_{n}(x) \in{ }^{e} \mathcal{L}_{n}$ are eigenvalues of the generalized eigenvalue problem (GEP)

$$
\mathbf{J}_{n} \vec{v}_{n, j}=x_{n, j} \mathbf{B}_{n} \vec{v}_{n, j}
$$


with

$$
\vec{v}_{n, j}=\eta_{n}\left\{\sum_{k=0}^{n-1}\left|\varphi_{k}\left(x_{n, j}\right)\right|^{2}\right\}^{-1 / 2} \vec{\varphi}_{n}\left(x_{n, j}\right), \quad\left|\eta_{n}\right|=1,
$$

the corresponding normalized eigenvector.

In the remainder of this paper we will assume that the sequence of nORFs $\left\{\varphi_{k}\right\}_{k=0}^{n}$ exists and that $\varphi_{k} \in{ }^{d e} \mathcal{L}_{k}$ for $k=0, \ldots, n .{ }^{1}$

3. Positive rational interpolatory quadrature rules. Consider the set of $n$ nodes $\left\{x_{n, k}^{\mathfrak{F}}\right\}_{k=1}^{n} \subset \mathbb{R}_{\mathcal{A}_{n}}$, where $\mathcal{A}_{n}=\left\{\alpha_{k}\right\}_{k=1}^{n}$ and $x_{n, k}^{\mathfrak{F}} \neq x_{n, j}^{\mathfrak{F}}$ if $k \neq j$, and weights $\left\{\lambda_{n, k}\right\}_{k=1}^{n} \subset \mathbb{R}_{0}^{+}$. We then call a quadrature formula of the form

$$
\mathfrak{F}_{n}\{f\}:=\sum_{k=1}^{n} \lambda_{n, k}^{\mathfrak{F}} f\left(x_{n, k}^{\mathfrak{F}}\right),
$$

an $n$th positive rational interpolatory quadrature formula (abbreviated PRIQ) iff $\mathfrak{R}_{n}\{f\}:=\mathfrak{F}\{f\}-\mathfrak{F}_{n}\{f\}=0$ for every $f \in \mathcal{R}_{n-1, \tilde{j}}=\mathcal{L}_{n-1} \cdot \tilde{\mathcal{L}}_{j}^{c}$, where $\mathcal{L}_{0} \subseteq \tilde{\mathcal{L}}_{j} \subset \mathcal{L}_{n+1}^{\left[\bar{\alpha}_{n}\right]}$. If $\mathcal{R}_{n-1, \tilde{j}}$ is the largest space for which $\mathfrak{R}_{n}\{f\}=0$, then it is called the domain of validity. Clearly, a necessary (but not sufficient) condition for the existence of a PRIQ is that $\mathcal{R}_{n-1, \tilde{j}}=\mathcal{R}_{n-1, \tilde{j}}^{c}$; i.e., for every function $f$ in the domain of validity it should hold that $f^{c}$ is in the domain of validity too. ${ }^{2}$

With ' $\left(n+i-1: n-i-m, \mathcal{A}_{n}, \mathfrak{F}\right)$-PRIQ', where $i \in\{0,1\}$ and $m \in\{1-i, \ldots, n-i\}$, we denote an $n$th PRIQ for which the space $\mathcal{R}_{n+i-1, n-i-m}$ is contained in the domain of validity (i.e., the quadrature is exact for at least every $f \in \mathcal{R}_{n+i-1, n-i-m}$ ). To study $\left(n+i-1: n-i-m, \mathcal{A}_{n}, \mathfrak{F}\right)$-PRIQs we will need the so-called quasi-orthogonal rational functions (qORFs), which are defined as follows.

Definition 3.1. Let $0 \leqslant m \leqslant n$. We then call a rational function $Q_{n, \vec{a}_{m+1}} \in \mathcal{L}_{n}$ an $m$-qORF of the first kind iff $Q_{n, \vec{a}_{m+1}}$ is of the form

$$
Q_{n, \vec{a}_{m+1}}(x)=\sum_{j=0}^{m} a_{j} \varphi_{n-j}(x), \quad \vec{a}_{m+1}=\left(\begin{array}{lll}
a_{0} & \ldots & a_{m}
\end{array}\right)^{T} \in \mathbb{C}^{m+1} .
$$

Definition 3.2. Let $0 \leqslant m \leqslant n$. We then call a rational function $P_{n, \vec{a}_{m+1}} \in \mathcal{L}_{n}$ an $m$-qORF of the second kind iff $P_{n, \vec{a}_{m+1}}$ is of the form

$P_{n, \vec{a}_{m+1}}(x)=a_{0} \varphi_{n}(x)+\sum_{j=1}^{m} a_{j} \frac{Z_{n}(x)}{Z_{n-j}^{c}(x)} \varphi_{n-j}(x), \quad \vec{a}_{m+1}=\left(\begin{array}{ccc}a_{0} & \ldots & a_{m}\end{array}\right)^{T} \in \mathbb{C}^{m+1}$.

From the previous definitions it clearly follows that $Q_{n, \vec{a}_{m+1}} \perp_{\mathfrak{F}} \mathcal{L}_{n-(m+1)}$ and that

$$
P_{n, \vec{a}_{m+1}} \perp_{\mathfrak{F}} \begin{cases}\mathcal{L}_{n-1}, & m=0 \\ \mathcal{L}_{n-m}\left(\bar{\alpha}_{n}\right) \equiv \frac{Z_{n-m}}{Z_{n}^{c}} \mathcal{L}_{n-(m+1)}, & 0<m<n \\ \mathcal{L}_{0}\left(\bar{\alpha}_{n}\right) \equiv \mathcal{L}_{-1}, & m=n\end{cases}
$$

\footnotetext{
${ }^{1}$ It is easily proved by induction over $k=1, \ldots, n$, with the aid of (2.5)-(2.6) and Theorem 2.1, that $\varphi_{k} \in{ }^{d e} \mathcal{L}_{k}$ for $k=0, \ldots, n$ iff $\left\{a_{k+1}^{[\varphi]}, c_{k}^{[\varphi]}\right\}_{k=0}^{n-2} \subset \mathbb{C}_{0},\left\{b_{k}^{[\varphi]}\right\}_{k=0}^{n-1} \subset \mathbb{C}$ and $\operatorname{det}\left\{\frac{1}{\alpha_{k}} \mathbf{J}_{k}-\mathbf{B}_{k}\right\} \neq 0$ for $k=1, \ldots, n$.

${ }^{2}$ If this condition is not satisfied, the weights cannot be all real; see [5, Sect. 2].
} 
Conversely, for every $r_{n} \in \mathcal{L}_{n}$ and every $m \in\{0, \ldots, n\}$ it holds that $r_{n} \perp_{\mathfrak{F}} \mathcal{L}_{n-(m+1)}$ iff $r_{n}$ is an $m$-qORF of the first kind. Further, assume that there exists a rational function $\varphi_{n}^{\left[\bar{\alpha}_{n-m}\right]} \in{ }^{e} \mathcal{L}_{n}^{\left[\bar{\alpha}_{n-m}\right]}$ so that $\varphi_{n}^{\left[\bar{\alpha}_{n-m}\right]} \perp_{\mathfrak{F}} \mathcal{L}_{n-1}$ and $\left\|\varphi_{n}^{\left[\bar{\alpha}_{n-m}\right]}\right\|_{\mathfrak{F}}=1$. Then it holds that $r_{n} \perp_{\mathfrak{F}} \mathcal{L}_{n-m}\left(\bar{\alpha}_{n}\right)$ iff $r_{n}$ is an $m$-qORF of the second kind. The first statement is trivial. To prove the second statement, we first need the following lemma.

Lemma 3.3. Let $1 \leqslant m \leqslant n$. Then for every sequence of constants $\left\{a_{j}\right\}_{j=1}^{m} \subset \mathbb{C}$ there exists a sequence of constants $\left\{b_{j}\right\}_{j=0}^{m+1} \subset \mathbb{C}$ so that

$$
Z_{n}(x) \sum_{j=1}^{m} a_{j} \varphi_{n-j}(x) \equiv b_{0} \varphi_{n}(x)+\sum_{j=1}^{m+1} b_{j} \frac{Z_{n}(x)}{Z_{n-j}^{c}(x)} \varphi_{n-j}(x) .
$$

Proof. For $m=1$ it follows from Theorem 2.1 that

$$
\frac{1}{E_{n}} \varphi_{n}(x)=Z_{n}(x)\left[1+\frac{D_{n}}{Z_{n-1}(x)}\right] \varphi_{n-1}(x)-C_{n} \frac{Z_{n}(x)}{Z_{n-2}^{c}(x)} \varphi_{n-2}(x),
$$

where it holds that

$$
1+\frac{D_{n}}{Z_{n-1}(x)}=\bar{E}_{n-1} C_{n}+\frac{D_{n}}{Z_{n-1}^{c}(x)} .
$$

Consequently, the statement follows for $m=1$ by taking $b_{0}=\frac{a_{1}}{E_{n} \bar{E}_{n-1} C_{n}} \in \mathbb{C}, b_{1}=$ $-\frac{a_{1} D_{n}}{\overline{E_{n-1} C_{n}}} \in \mathbb{C}$ and $b_{2}=\frac{a_{1}}{\bar{E}_{n-1}} \in \mathbb{C}$.

So, suppose now that the statement holds true for $1 \leqslant m-1<n$. For $m$ we then have that

$$
\begin{aligned}
Z_{n}(x) \sum_{j=1}^{m} a_{j} \varphi_{n-j}(x)=Z_{n}(x) \sum_{j=1}^{m-1} a_{j} \varphi_{n-j}(x)+a_{m} Z_{n}(x) \varphi_{n-m}(x) \equiv \\
b_{0} \varphi_{n}(x)+\sum_{j=1}^{m} b_{j} \frac{Z_{n}(x)}{Z_{n-j}^{c}(x)} \varphi_{n-j}(x)+a_{m} Z_{n}(x) \varphi_{n-m}(x) .
\end{aligned}
$$

From Theorem 2.1 it now follows that

$$
\begin{aligned}
& \frac{1}{E_{n-(m-1)}} \frac{Z_{n}(x)}{Z_{n-(m-1)}(x)} \varphi_{n-(m-1)}(x)= \\
& \quad Z_{n}(x)\left[1+\frac{D_{n-(m-1)}}{Z_{n-m}(x)}\right] \varphi_{n-m}(x)-C_{n-(m-1)} \frac{Z_{n}(x)}{Z_{n-(m+1)}^{c}(x)} \varphi_{n-(m+1)}(x),
\end{aligned}
$$

where it holds that

$$
\begin{aligned}
1+\frac{D_{n-(m-1)}}{Z_{n-m}(x)}=\bar{E}_{n-m} C_{n-(m-1)}+\frac{D_{n-(m-1)}}{Z_{n-m}^{c}(x)} \\
\quad \text { and } \frac{1}{Z_{n-(m-1)}(x)}=\frac{1}{Z_{n-(m-1)}^{c}(x)}-\frac{1}{Z_{n-(m-1)}^{c}\left(\alpha_{n-(m-1)}\right)} .
\end{aligned}
$$

As a result we find that

$$
a_{m} Z_{n}(x) \varphi_{n-m}(x)=\sum_{j=m-1}^{m+1} d_{j} \frac{Z_{n}(x)}{Z_{n-j}^{c}(x)} \varphi_{n-j}(x)+e_{m} Z_{n}(x) \varphi_{n-(m-1)}(x),
$$


with $d_{m-1}=\frac{a_{m}}{E_{n-(m-1)} \bar{E}_{n-m} C_{n-(m-1)}} \in \mathbb{C}, d_{m}=-\frac{a_{m} D_{n-(m-1)}}{\bar{E}_{n-m} C_{n-(m-1)}} \in \mathbb{C}, d_{m+1}=$ $\frac{a_{m}}{\bar{E}_{n-m}} \in \mathbb{C}$ and $e_{m}=-\frac{a_{m}}{E_{n-(m-1)} \bar{E}_{n-m} C_{n-(m-1)} Z_{n-(m-1)}^{c}\left(\alpha_{n-(m-1)}\right)} \in \mathbb{C}$. Finally, from the induction hypothesis it follows that there exist constants $\left\{\hat{c}_{j}\right\}_{j=0}^{m} \subset \mathbb{C}$ so that

$$
Z_{n}(x) \varphi_{n-(m-1)}(x) \equiv \hat{c}_{0} \varphi_{n}(x)+\sum_{j=1}^{m} \hat{c}_{j} \frac{Z_{n}(x)}{Z_{n-j}^{c}(x)} \varphi_{n-j}(x) .
$$

This ends the proof. $\square$

We are now able to prove the following.

TheOREM 3.4. Let $1 \leqslant m \leqslant n$, and suppose $r_{n} \in \mathcal{L}_{n}$. Further, assume that

$$
\varphi_{n}^{\left[\bar{\alpha}_{n-m}\right]} \in{ }^{e} \mathcal{L}_{n}^{\left[\bar{\alpha}_{n-m}\right]}: \varphi_{n}^{\left[\bar{\alpha}_{n-m}\right]} \perp_{\mathfrak{F}} \mathcal{L}_{n-1} \text { and }\left\|\varphi_{n}^{\left[\bar{\alpha}_{n-m}\right]}\right\|_{\mathfrak{F}}=1 .
$$

Then it then holds that $r_{n} \perp_{\mathfrak{F}} \mathcal{L}_{n-m}\left(\bar{\alpha}_{n}\right)$ iff $r_{n}$ is an $m-q O R F$ of the second kind.

Proof. From the definition of an $m$-qORF of the second kind it is clear that $r_{n} \perp_{\mathfrak{F}} \mathcal{L}_{n-m}\left(\bar{\alpha}_{n}\right)$ if $r_{n}$ is an $m$-qORF of the second kind. So, we only need to prove that $r_{n}$ is an $m$-qORF of the second kind if $r_{n} \perp_{\mathfrak{F}} \mathcal{L}_{n-m}\left(\bar{\alpha}_{n}\right)$.

The case in which $m=1$ has already been proved in [6, Thm. 5] under the less restrictive condition that $\varphi_{n}^{\left[\bar{\alpha}_{n-1}\right]} \in \mathcal{L}_{n}^{\left[\bar{\alpha}_{n-1}\right]} \backslash \mathcal{L}_{n-1}$. Thus, suppose that $1<m \leqslant n$. Since $r_{n} \perp_{\mathfrak{F}} \mathcal{L}_{n-m}\left(\bar{\alpha}_{n}\right)$, it follows that $\frac{Z_{n-m}^{c}}{Z_{n}} r_{n} \in \mathcal{L}_{n}^{\left[\bar{\alpha}_{n-m}\right]}$ and $\frac{Z_{n-m}^{c}}{Z_{n}} r_{n} \perp_{\mathfrak{F}} \mathcal{L}_{n-(m+1)}$. Consider now the nORF $\varphi_{n}^{\left[\bar{\alpha}_{n-m}\right]} \in{ }^{e} \mathcal{L}_{n}^{\left[\bar{\alpha}_{n-m}\right]}$. Then there exist constants $\left\{b_{j}\right\}_{j=0}^{m} \subset \mathbb{C}$ so that

$$
\frac{Z_{n-m}^{c}(x)}{Z_{n}(x)} r_{n}(x)=b_{0} \varphi_{n}^{\left[\bar{\alpha}_{n-m}\right]}(x)+\sum_{j=1}^{m} b_{j} \varphi_{n-j}(x)
$$

and hence,

$$
\begin{gathered}
r_{n}(x)=b_{0} \frac{Z_{n}(x)}{Z_{n-m}^{c}(x)} \varphi_{n}^{\left[\bar{\alpha}_{n-m}\right]}(x)+\sum_{j=1}^{m} b_{j} \frac{Z_{n}(x)}{Z_{n-m}^{c}(x)} \varphi_{n-j}(x)= \\
b_{0} \frac{Z_{n}(x)}{Z_{n-m}^{c}(x)} \varphi_{n}^{\left[\bar{\alpha}_{n-m}\right]}(x)+Z_{n}(x) \sum_{j=1}^{m-1} \frac{b_{j}}{Z_{n-m}^{c}\left(\bar{\alpha}_{n-j}\right)} \varphi_{n-j}(x)+\sum_{j=1}^{m} b_{j} \frac{Z_{n}(x)}{Z_{n-j}^{c}(x)} \varphi_{n-j}(x) .
\end{gathered}
$$

From the previous lemma it now follows that there exist constants $\left\{c_{j}\right\}_{j=0}^{m} \subset \mathbb{C}$ so that

$$
r_{n}(x)=b_{0} \frac{Z_{n}(x)}{Z_{n-m}^{c}(x)} \varphi_{n}^{\left[\bar{\alpha}_{n-m}\right]}(x)+c_{0} \varphi_{n}(x)+\sum_{j=1}^{m} c_{j} \frac{Z_{n}(x)}{Z_{n-j}^{c}(x)} \varphi_{n-j}(x) .
$$

Thus, it remains to prove that $\frac{Z_{n}}{Z_{n-m}^{c}} \varphi_{n}^{\left[\bar{\alpha}_{n-m}\right]}$ is an $m$-qORF of the second kind. Since $\varphi_{n}^{\left[\bar{\alpha}_{n-m}\right]} \in{ }^{e} \mathcal{L}_{n}^{\left[\bar{\alpha}_{n-m}\right]}$, we deduce from Theorem 2.1 that

$$
\begin{gathered}
\frac{1}{E_{n}^{\left[\bar{\alpha}_{n-m}\right]}} \frac{Z_{n}(x)}{Z_{n-m}^{c}(x)} \varphi_{n}^{\left[\bar{\alpha}_{n-m}\right]}(x)= \\
Z_{n}(x)\left[1+\frac{D_{n}^{\left[\bar{\alpha}_{n-m}\right]}}{Z_{n-1}(x)}\right] \varphi_{n-1}(x)-C_{n}^{\left[\bar{\alpha}_{n-m}\right]} \frac{Z_{n}(x)}{Z_{n-2}^{c}(x)} \varphi_{n-2}(x)= \\
\bar{E}_{n-1} C_{n}^{\left[\bar{\alpha}_{n-m}\right]} Z_{n}(x) \varphi_{n-1}(x)+D_{n}^{\left[\bar{\alpha}_{n-m}\right]} \frac{Z_{n}(x)}{Z_{n-1}^{c}(x)} \varphi_{n-1}(x)-C_{n}^{\left[\bar{\alpha}_{n-m}\right]} \frac{Z_{n}(x)}{Z_{n-2}^{c}(x)} \varphi_{n-2}(x) .
\end{gathered}
$$


Finally, because $Z_{n} \varphi_{n-1}$ is a $2-q \mathrm{ORF}$ of the second kind due to the previous lemma, it follows that $\frac{Z_{n}}{Z_{n-m}^{c}} \varphi_{n}^{\left[\bar{\alpha}_{n-m}\right]}$ is an $m$-qORF of the second kind. This concludes the proof. $\mathrm{T}$

In everything that follows, we will assume that the $\operatorname{nORF} \varphi_{n}^{\left[\bar{\alpha}_{n-m}\right]}$ in (3.2) exists. The following two theorems now give the connection between $m$-qORFs and $(n$ : $\left.n-1-m, \mathcal{A}_{n}, \mathfrak{F}\right)$-PRIQs, where $i \in\{0,1\}$ and $m \in\{1-i, \ldots, n-i\}$.

TheOREM 3.5. Let $0 \leqslant m \leqslant n-1$, and consider $a\left(n: n-1-m, \mathcal{A}_{n}, \mathfrak{F}\right)-P R I Q$ of the form (3.1). Then there exists an $m$-qORF of the first kind $Q_{n, \vec{a}_{m+1}} \in{ }^{r} \mathcal{L}_{n}$ so that $Q_{n, \vec{a}_{m+1}}\left(x_{n, k}^{\mathfrak{F}}\right)=0$ for $k=1, \ldots, n$, and

$$
\lambda_{n, k}^{\mathfrak{F}}=\mathfrak{F}\left\{L_{n, k}^{[Q]}\right\}, \quad k=1, \ldots, n,
$$

with $L_{n, k}^{[Q]}$ the fundamental interpolating rational functions defined by

$$
L_{n, k}^{[Q]}(x)=\frac{\left(1-x / \alpha_{n}\right)}{\left(1-x_{n, k}^{\mathfrak{F}} / \alpha_{n}\right)} \cdot \frac{Q_{n, \vec{a}_{m+1}}(x)}{\left(x-x_{n, k}^{\mathfrak{F}}\right) Q_{n, \vec{a}_{m+1}}^{\prime}\left(x_{n, k}^{\mathfrak{F}}\right)} \in \mathcal{L}_{n-1}, \quad k=1, \ldots, n,
$$

where the prime denotes the derivative with respect to $x$.

Proof. (The proof is similar to the proof of [1, Thm. 11.6.1].) First, note that $L_{n, k}^{[Q]}\left(x_{n, j}\right)=\delta_{k, j}, 1 \leqslant k, j \leqslant n$. Consider now the auxiliary function

$$
h(x)=f(x)-\sum_{k=1}^{n} f\left(x_{n, k}\right) \cdot L_{n, k}^{[Q]}(x) .
$$

For $f \in \mathcal{R}_{n, n-1-m}$, this can be written as

$$
h(x)=\frac{p_{2 n-1-m}(x)}{\pi_{n}(x) \cdot \pi_{n-1-m}^{c}(x)}, \quad p_{2 n-1-m} \in \mathcal{P}_{2 n-1-m} .
$$

Since $h\left(x_{n, k}\right)=0$ for $k=1, \ldots, n$ (interpolation property), and since the numerator of $Q_{n, \vec{a}_{m+1}}$ is a nonzero constant times the nodal polynomial $\left(x-x_{n, 1}\right) \cdots\left(x-x_{n, n}\right)$, we can write

$$
h(x)=Q_{n, \vec{a}_{m+1}}(x) \cdot g^{c}(x), \quad g \in \mathcal{L}_{n-1-m} .
$$

From (3.4), together with (3.1) and (3.3), it now follows that

$$
\mathfrak{F}\{h\}=\mathfrak{F}\left\{Q_{n, \vec{a}_{m+1}} \cdot g^{c}\right\}=\mathfrak{R}_{n}\{f\} .
$$

Finally, since the quadrature is exact for every $f \in \mathcal{R}_{n, n-1-m}$ (i.e., $\mathfrak{R}_{n}\{f\}=0$ for every $\left.f \in \mathcal{R}_{n, n-1-m}\right)$, it follows that $Q_{n, \vec{a}_{m+1}}$ is an $m$-qORF of the first kind. This ends the proof. $\square$

THEOREM 3.6. Let $1 \leqslant m \leqslant n$, and consider a $\left(n-1: n-m, \mathcal{A}_{n}, \mathfrak{F}\right)-P R I Q$ of the form (3.1). Then there exists an $m$-qORF of the second kind $P_{n, \vec{a}_{m+1}} \in{ }^{r} \mathcal{L}_{n}$ so that $P_{n, \vec{a}_{m+1}}\left(x_{n, k}^{\mathfrak{F}}\right)=0$ for $k=1, \ldots, n$, and

$$
\lambda_{n, k}^{\mathfrak{F}}=\mathfrak{F}\left\{L_{n, k}^{[P]}\right\}, \quad k=1, \ldots, n,
$$

with $L_{n, k}^{[P]}$ the fundamental interpolating rational functions defined by

$$
L_{n, k}^{[P]}(x)=\frac{\left(1-x / \alpha_{n}\right)}{\left(1-x_{n, k}^{\mathfrak{F}} / \alpha_{n}\right)} \cdot \frac{P_{n, \vec{a}_{m+1}}(x)}{\left(x-x_{n, k}^{\mathfrak{F}}\right) P_{n, \vec{a}_{m+1}}^{\prime}\left(x_{n, k}^{\mathfrak{F}}\right)} \in \mathcal{L}_{n-1}, \quad k=1, \ldots, n,
$$


where the prime denotes the derivative with respect to $x$.

Proof. (The proof is similar as above.) First, note that $L_{n, k}^{[P]}\left(x_{n, j}\right)=\delta_{k, j}, 1 \leqslant$ $k, j \leqslant n$. Consider the auxiliary function

$$
h(x)=f(x)-\sum_{k=1}^{n} f\left(x_{n, k}\right) \cdot L_{n, k}^{[P]}(x) .
$$

For $f \in \mathcal{R}_{n-1, n-m}$, this can be written as

$$
h(x)=\frac{p_{2 n-1-m}(x)}{\pi_{n-1}(x) \cdot \pi_{n-m}^{c}(x)}, \quad p_{2 n-1-m} \in \mathcal{P}_{2 n-1-m} .
$$

Since $h\left(x_{n, k}\right)=0$ for $k=1, \ldots, n$ (interpolation property), and since the numerator of $P_{n, \vec{a}_{m+1}}$ is a nonzero constant times the nodal polynomial $\left(x-x_{n, 1}\right) \cdots\left(x-x_{n, n}\right)$, we can write

$$
h(x)=P_{n, \vec{a}_{m+1}}(x) \cdot g^{c}(x),
$$

where

$$
g(x)=\frac{\left(1-x / \bar{\alpha}_{n}\right) p_{n-1-m}(x)}{\pi_{n-m}^{c}(x)}, \quad p_{n-1-m} \in \mathcal{P}_{n-1-m} .
$$

Hence, $g \in \mathcal{L}_{n-m}\left(\bar{\alpha}_{n}\right)$. From (3.6), together with (3.1) and (3.5), it follows that

$$
\mathfrak{F}\{h\}=\mathfrak{F}\left\{P_{n, \vec{a}_{m+1}} \cdot g^{c}\right\}=\mathfrak{R}_{n}\{f\} .
$$

Finally, since the quadrature is exact for every $f \in \mathcal{R}_{n-1, n-m}$ (i.e., $\mathfrak{R}_{n}\{f\}=0$ for every $\left.f \in \mathcal{R}_{n-1, n-m}\right)$, it follows that $P_{n, \vec{a}_{m+1}}$ is an $m$-qORF of the second kind. This ends the proof.

4. Existence of rational Gauss-type quadrature rules. The rational Gaussian quadrature rule is a special kind of PRIQ that has maximal domain of validity $\mathcal{R}_{n, n-1}$; hence, it is a $\left(n+i-1: n-i-m, \mathcal{A}_{n}, \mathfrak{F}\right)$-PRIQ with $i=1$ and $m=0$. It is well known that the rational Gaussian quadrature rule exists iff $\alpha_{n} \in \overline{\mathbb{R}}_{0}$ and $\varphi_{n} \in{ }^{r} \mathcal{L}_{n}$. Whenever it exists, it is unique, and the nodes can be computed by solving the GEP (2.7), while the corresponding weights can be found by means of the corresponding eigenvectors as follows:

$$
\lambda_{n, k}^{\mathfrak{F}}=\left|v_{n, k}\right|^{2}\|1\|_{\mathfrak{F}}^{2},
$$

where $v_{n, k}$ denotes the first entry in the normalized eigenvector $\vec{v}_{n, k}$. See e.g. [6, Sect. 5].

From the previous two theorems we can deduce that $m$-qORFs, with $1 \leqslant m \leqslant n-i$ and $i \in\{0,1\}$, can be used to construct $\left(n+i-1: n-i-m, \mathcal{A}_{n}, \mathfrak{F}\right)$-PRIQs with $m$ nodes fixed in advance. Indeed, consider an $n$th PRIQ of the form

$$
\mathfrak{F}_{n}\{f\}=\sum_{k=1}^{m} \lambda_{n, k}^{\mathfrak{F}} f\left(x_{n, k}^{\mathfrak{F}}\right)+\sum_{k=m+1}^{n} \lambda_{n, k}^{\mathfrak{F}} f\left(x_{n, k}^{\mathfrak{F}}\right),
$$

where the sequence of $m$ distinct nodes $\mathcal{X}_{m}=\left\{x_{n, k}^{\widetilde{F}}\right\}_{k=1}^{m} \subset \mathbb{R}_{\mathcal{A}_{n}}$ are fixed in advance, and the remaining nodes and corresponding weights are chosen such that the PRIQ is 
a $\left(n+i-1: n-i-m, \mathcal{A}_{n}, \mathfrak{F}\right)$-PRIQ. Then these remaining nodes and corresponding weights can be found by means of the $m$-qORF $Q_{n, \vec{a}_{m+1}}$ (for $i=1$ ) or $P_{n, \vec{a}_{m+1}}$ (for $i=0$ ), with the coefficients $\vec{a}_{m+1}$ chosen in such a way that the $m$-qORF has zeros in $\mathcal{X}_{m}$. Clearly, the existence of the $\left(n+i-1: n-i-m, \mathcal{A}_{n}, \mathfrak{F}\right)$-PRIQ $(4.2)$ depends on the sequence of poles $\mathcal{A}_{n+i-1}$ and the sequence of nodes $\mathcal{X}_{m}$ (i.e., on whether the zeros of the $m$-qORF are all distinct real, and the corresponding weights are positive, for the given sequences $\mathcal{A}_{n+i-1}$ and $\mathcal{X}_{m}$ ).

A special kind of PRIQs with nodes fixed in advance are the rational GaussRadau $(m=1)$ and Gauss-Lobatto $(m=2)$ quadrature rules. The existence of rational Gauss-Radau quadrature rules has been studied in $[6$, Sect. 5] for the case of $i=0$. In this reference it has been proved that, if $P_{n, \vec{a}_{2}} \in{ }^{d} \mathcal{L}_{n}$, there exist HPD linear functionals $\mathfrak{G}$, for which $\left\{\varphi_{j}\right\}_{j=0}^{n-1}$ forms an orthonormal system in $\mathcal{L}_{n-1}$ with respect to the inner product $\langle f, g\rangle_{\mathfrak{G}}=\mathfrak{G}\left\{f g^{c}\right\}$, and poles $\alpha \in \overline{\mathbb{C}}_{0}$, so that $\frac{Z_{\alpha}}{Z_{n}} P_{n, \vec{a}_{2}} \perp_{\mathfrak{G}} \mathcal{L}_{n-1}$. For this reason, whenever a $\left(n-1: n-1, \mathcal{A}_{n}, \mathfrak{F}\right)$-PRIQ exists, it is a $\left(n: n-1,\left\{\alpha_{1}, \ldots, \alpha_{n-1}, \alpha\right\}, \mathfrak{G}\right)$-PRIQ (i.e., a rational Gaussian quadrature rule) too. Consequently, its nodes can be computed by solving a modified GEP of the form (2.7), with the coefficients $a_{n-1}^{[\varphi]}$ and $b_{n-1}^{[\varphi]}$ that appear in the last row of the matrices $\mathbf{J}_{n}$ and $\mathbf{S}_{n}$ replaced with $a_{n-1, x_{n, 1}}^{[P]}$ and $b_{n-1, x_{n, 1}}^{[P]}$ (depending on the fixed node; see [6, Sect. 3]), while the corresponding weights again can be found by means of the corresponding eigenvectors.

The aim of this section now is to study the existence and construction of the rational Gauss-Radau quadrature rules for $i=1$, and the rational Gauss-Lobatto quadrature rules for $i=0$.

4.1. Rational Gauss-Radau quadrature rules for $i=1$. Recall from Section 3 that a necessary (but not sufficient) condition for the existence of a PRIQ is that for every function $f$ in the domain of validity it should hold that $f^{c}$ is in the domain of validity too. Since a $\left(n: n-2, \mathcal{A}_{n}, \mathfrak{F}\right)$-PRIQ should at least be exact for every $f \in \mathcal{R}_{n, n-2}$, it follows that it should also be exact for every $f \in \mathcal{R}_{n-2, n}$, and hence, for every $f \in \mathcal{R}_{n-2, n-2} \cdot \mathcal{S}\left\{\alpha_{n-1}, \alpha_{n}\right\}$. This leaves us with the following possible situations:

1. If $\left\{\alpha_{n-1}, \alpha_{n}\right\} \subset \mathbb{C}_{\mathbb{R}}$, then

(a) for $\alpha_{n}=\bar{\alpha}_{n-1}$ it follows that $\mathcal{R}_{n-2, n-2} \cdot \mathcal{S}\left\{\alpha_{n-1}, \alpha_{n}\right\}=\mathcal{R}_{n-1, n-1}$ $\left(=\mathcal{R}_{n, n-2}\right)$, so that the $\left(n: n-2, \mathcal{A}_{n}, \mathfrak{F}\right)$-PRIQ is a $\left(n-1: n-1, \mathcal{A}_{n}, \mathfrak{F}\right)$ PRIQ (i.e., a rational Gauss-Radau quadrature rule with $i=0$ ) too;

(b) for $\alpha_{n} \neq \bar{\alpha}_{n-1}$ it follows that $\mathcal{R}_{n-2, n-2} \cdot \mathcal{S}\left\{\alpha_{n-1}, \alpha_{n}\right\}=\mathcal{R}_{n, n}$, so that the $\left(n: n-2, \mathcal{A}_{n}, \mathfrak{F}\right)$-PRIQ does not exist.

2. If $\alpha_{n-1} \in \mathbb{C}_{\mathbb{R}}$ and $\alpha_{n} \in \overline{\mathbb{R}}_{0}$, it follows that $\mathcal{R}_{n-2, n-2} \cdot \mathcal{S}\left\{\alpha_{n-1}, \alpha_{n}\right\}=\mathcal{R}_{n, n-1}$ $\left(\supset \mathcal{R}_{n, n-2}\right)$, so that the $\left(n: n-2, \mathcal{A}_{n}, \mathfrak{F}\right)$-PRIQ is a $\left(n: n-1, \mathcal{A}_{n}, \mathfrak{F}\right)-$ PRIQ (i.e., the rational Gaussian quadrature rule) too.

3. If $\alpha_{n-1} \in \overline{\mathbb{R}}_{0}$ and $\alpha_{n} \in \mathbb{C}_{\mathbb{R}}$, it follows that $\mathcal{R}_{n-2, n-2} \cdot \mathcal{S}\left\{\alpha_{n-1}, \alpha_{n}\right\}=$ $\mathcal{R}_{n, n-2} \cdot \mathcal{L}\left\{\bar{\alpha}_{n}\right\}\left(\supset \mathcal{R}_{n, n-2}\right)$, so that the $\left(n: n-2, \mathcal{A}_{n}, \mathfrak{F}\right)$-PRIQ is a $(n:$ $\left.n-1,\left\{\alpha_{1}, \ldots, \alpha_{n-2}, \alpha_{n}, \alpha_{n-1}\right\}, \mathfrak{F}\right)$-PRIQ (i.e., the rational Gaussian quadrature rule for the case in which the last two poles are interchanged) too.

4. If $\left\{\alpha_{n-1}, \alpha_{n}\right\} \subset \overline{\mathbb{R}}_{0}$, then

(a) for $\alpha_{n}=\alpha_{n-1}$ it follows that $\mathcal{R}_{n-2, n-2} \cdot \mathcal{S}\left\{\alpha_{n-1}, \alpha_{n}\right\}=\mathcal{R}_{n-1, n-1}$ $\left(=\mathcal{R}_{n, n-2}\right)$, so that the $\left(n: n-2, \mathcal{A}_{n}, \mathfrak{F}\right)$-PRIQ is a $\left(n-1: n-1, \mathcal{A}_{n}, \mathfrak{F}\right)$ PRIQ (i.e., a rational Gauss-Radau quadrature rule with $i=0$ ) too;

(b) for $\alpha_{n} \neq \alpha_{n-1}$ it follows that $\mathcal{R}_{n-2, n-2} \cdot \mathcal{S}\left\{\alpha_{n-1}, \alpha_{n}\right\}=\mathcal{R}_{n, n-2}$. 
For the first two situations (1. and 2.), the existence of the rational Gauss-Radau quadrature rule (with $i=1$ ) can easily be verified by checking the existence of the corresponding rational Gauss-Radau quadrature rule (with $i=0$ ) or rational Gaussian quadrature rule for the given sequence of poles $\mathcal{A}_{n}$ and fixed node $x_{n, 1}^{\mathfrak{F}}$. Thus, in what follows we can restrict ourselves to the case in which $\alpha_{n-1} \in \overline{\mathbb{R}}_{0}$ and $\alpha_{n} \in \overline{\mathbb{C}}_{\left\{0, \alpha_{n-1}\right\}} \cdot{ }^{3}$ We then have the following lemma.

LEMMA 4.1. A 1-qORF of the first kind

$$
Q_{n, \vec{a}_{2}}(x)=a_{0} \varphi_{n}(x)+a_{1} \varphi_{n-1}(x) \in{ }^{d e} \mathcal{L}_{n}
$$

satisfies a relation of the form

$$
Q_{n, \vec{a}_{2}}(x)=E_{n}^{[Q]} Z_{n}(x)\left\{\left[1+\frac{D_{n}^{[Q]}}{Z_{n-1}(x)}\right] K_{n} \varphi_{n-1}(x)-\frac{C_{n}^{[Q]}}{Z_{n-2}^{c}(x)} \varphi_{n-2}(x)\right\}
$$

where $K_{n} \in \mathbb{C}_{0}$,

$$
\begin{aligned}
E_{n}^{[Q]} & =\frac{a_{0} E_{n}+a_{1} / Z_{n}\left(\alpha_{n-1}\right)}{K_{n}} \in \mathbb{C}_{0}, \quad D_{n}^{[Q]}=\frac{a_{0} E_{n} D_{n}+a_{1}}{a_{0} E_{n}+a_{1} / Z_{n}\left(\alpha_{n-1}\right)}, \\
C_{n}^{[Q]} & =\frac{\left|K_{n}\right|^{2} a_{0} E_{n}}{\bar{E}_{n-1}^{[Q]}\left[a_{0} E_{n}+a_{1} / Z_{n}\left(\alpha_{n-1}\right)\right]} \in \mathbb{C}_{0}, \quad \text { and } \quad E_{n-1}^{[Q]}=K_{n} E_{n-1} \in \mathbb{C}_{0} .
\end{aligned}
$$

Proof. From Theorem 2.1 it follows that

$$
\begin{aligned}
& Q_{n, \vec{a}_{2}}(x)= \\
& a_{0} E_{n} Z_{n}(x)\left\{\left[1+\frac{D_{n}}{Z_{n-1}(x)}\right] \varphi_{n-1}(x)-\frac{1}{\bar{E}_{n-1} Z_{n-2}^{c}(x)} \varphi_{n-2}(x)\right\}+a_{1} \varphi_{n-1}(x),
\end{aligned}
$$

where $a_{0} \neq 0$ due to $Q_{n, \vec{a}_{2}} \in \mathcal{L}_{n} \backslash \mathcal{L}_{n-1}$. Setting

$$
\varphi_{n-1}(x)=\frac{Z_{n}(x)}{Z_{n}(x)} \varphi_{n-1}(x)=Z_{n}\left[\frac{1}{Z_{n}\left(\alpha_{n-1}\right)}+\frac{1}{Z_{n-1}(x)}\right] \varphi_{n-1}(x),
$$

we obtain that

$$
Q_{n, \vec{a}_{2}}(x)=Z_{n}(x)\left\{\left[\tilde{E}_{n}+\frac{\tilde{D}_{n}}{Z_{n-1}(x)}\right] \varphi_{n-1}(x)-\frac{\tilde{C}_{n}}{Z_{n-2}^{c}(x)} \varphi_{n-2}(x)\right\},
$$

where

$$
\tilde{E}_{n}=a_{0} E_{n}+\frac{a_{1}}{Z_{n}\left(\alpha_{n-1}\right)}, \quad \tilde{D}_{n}=a_{0} E_{n} D_{n}+a_{1} \quad \text { and } \quad \tilde{C}_{n}=\frac{a_{0} E_{n}}{\bar{E}_{n-1}}
$$

Further, for $Q_{n, \vec{a}_{2}}=\frac{q_{n}}{\pi_{n}} \in{ }^{d e} \mathcal{L}_{n}$ we find with $\varphi_{k}=\frac{p_{k}}{\pi_{k}}$ that

$$
q_{n}\left(\alpha_{n-1}\right)=\tilde{E}_{n} \alpha_{n-1} p_{n-1}\left(\alpha_{n-1}\right) \neq 0,
$$

\footnotetext{
${ }^{3}$ Because of the re-ordering of the last two poles in the rational Gaussian quadrature rule for the case in which $\alpha_{n-1} \in \overline{\mathbb{R}}_{0}$ and $\alpha_{n} \in \mathbb{C}_{\mathbb{R}}$, we will allow $\alpha_{n}$ to be arbitrary complex.
} 
so that $\tilde{E}_{n} \neq 0$. The relation now follows by setting $\tilde{E}_{n}=E_{n}^{[Q]} K_{n}$ (for an arbitrary $\left.K_{n} \in \mathbb{C}_{0}\right), \tilde{D}_{n}=D_{n}^{[Q]} E_{n}^{[Q]} K_{n}$, and $\tilde{C}_{n}=E_{n}^{[Q]} C_{n}^{[Q]}$. प

As a consequence of the previous lemma and Theorem 2.2, we now can prove the following.

Lemma 4.2. Consider a 1-qORF of the first kind

$$
Q_{n, \vec{a}_{2}}(x)=a_{0} \varphi_{n}(x)+a_{1} \varphi_{n-1}(x)=\frac{q_{n}(x)}{\pi_{n}(x)} \in{ }^{d e} \mathcal{L}_{n} .
$$

Let $\alpha \in \overline{\mathbb{C}}_{0}$ be chosen in such a way that $q_{n}(\alpha) \neq 0$ and

$$
\mathfrak{T}\{\alpha\}=k \cdot\left(\mathcal{I} m\left\{a_{1} \bar{E}_{n} / a_{0}\right\}+\mathfrak{T}\left\{\alpha_{n}\right\}\right)
$$

for some $k \in \mathbb{R}_{0}^{+}$. Then there exist HPD linear functionals $\mathfrak{G}$, for which $\left\{\varphi_{j}\right\}_{j=0}^{n-2}$ forms an orthonormal system in $\mathcal{L}_{n-2}$ and $\varphi_{n-1} \perp_{\mathfrak{G}} \mathcal{L}_{n-2}$ with respect to the inner product defined by $\langle f, g\rangle_{\mathfrak{G}}=\mathfrak{G}\left\{f g^{c}\right\}$, so that $\frac{Z_{\alpha}}{Z_{n}} Q_{n, \vec{a}_{2}} \in{ }^{d e} \mathcal{L}_{n}^{[\alpha]}$ and $\frac{Z_{\alpha}}{Z_{n}} Q_{n, \vec{a}_{2}} \perp_{\mathfrak{G}} \mathcal{L}_{n-1}$ iff $\chi_{n}^{[Q]}:=\frac{a_{1}}{a_{0} E_{n}}\left(\frac{1}{\alpha_{n-1}}-\frac{1}{\alpha_{n}}\right) \in(-1, \infty)$.

Proof. Since $\alpha_{n-1} \in \overline{\mathbb{R}}_{0}$, it follows from assumptions (A4)-(A5) in Theorem 2.2 that for every $K_{n} \in \mathbb{C}_{0}$, there exist HPD linear functionals $\mathfrak{G}$ so that $K_{n} \varphi_{n-1}$ is a nORF in ${ }^{d e} \mathcal{L}_{n-1}$ with respect to the inner product defined by $\langle f, g\rangle_{\tilde{\mathfrak{G}}}=\tilde{\mathfrak{G}}\left\{f g^{c}\right\}$. So, let $\varphi_{n}^{[\alpha]}$ be given by

$$
\begin{aligned}
& \varphi_{n}^{[\alpha]}=\frac{E_{n}^{[\alpha]}}{E_{n}^{[Q]}} \frac{Z_{\alpha}(x)}{Z_{n}(x)} Q_{n, \vec{a}_{2}}(x)= \\
& E_{n}^{[\alpha]} Z_{\alpha}(x)\left\{\left[1+\frac{D_{n}^{[Q]}}{Z_{n-1}(x)}\right] K_{n} \varphi_{n-1}(x)-\frac{C_{n}^{[Q]}}{Z_{n-2}^{c}(x)} \varphi_{n-2}(x)\right\},
\end{aligned}
$$

where $E_{n}^{[\alpha]} \in \mathbb{C}_{0}$. Then it holds that $\varphi_{n}^{[\alpha]}$ satisfies assumptions (A1)-(A4) in Theorem 2.2. From assumption (A6) it now follows that $C_{n}^{[Q]}$ should be equal to $\left(\bar{K}_{n} \bar{E}_{n-1}\right)^{-1}$. From the previous lemma we deduce that this can only be for $\left|K_{n}\right|^{2}=1+\chi_{n}^{[Q]}$, which implies that $\chi_{n}^{[Q]}$ should be in $(-1, \infty)$. Finally, we have that

$$
\begin{array}{r}
\mathcal{I} m\left\{D_{n}^{[Q]}\right\}=\frac{\mathcal{I} m\left\{D_{n}\right\}+\mathcal{I} m\left\{\frac{a_{1}}{a_{0} E_{n}}\right\}}{1+\chi_{n}^{[Q]}}=\frac{\operatorname{Im}\left\{a_{1} \bar{E}_{n} / a_{0}\right\}+\mathfrak{T}\left\{\alpha_{n}\right\}}{\left(1+\chi_{n}^{[Q]}\right)\left|E_{n}\right|^{2}}-\frac{\mathfrak{T}\left\{\alpha_{n-2}\right\}}{\left|E_{n-1}^{[Q]}\right|^{2}}= \\
\frac{\mathfrak{T}\{\alpha\}}{k\left(1+\chi_{n}^{[Q]}\right)\left|E_{n}\right|^{2}}-\frac{\mathfrak{T}\left\{\alpha_{n-2}\right\}}{\left|E_{n-1}^{[Q]}\right|^{2}}
\end{array}
$$

where the second equality follows from assumption (A5) applied on $D_{n}$. Consequently, assumption (A5) in Theorem 2.2 is satisfied too for every $E_{n}^{[\alpha]} \in \mathbb{C}_{0}$ if $\mathfrak{T}\{\alpha\}=0$, respectively for $\left|E_{n}^{[\alpha]}\right|^{2}=k\left(1+\chi_{n}^{[Q]}\right)\left|E_{n}\right|^{2} \in \mathbb{R}_{0}^{+}$if $\mathfrak{T}\{\alpha\} \neq 0$.

The previous lemma now allows us to prove our first main result.

TheOREM 4.3. Consider a quadrature formula of the form

$$
\mathfrak{F}_{n}\{f\}=\lambda_{n, 1}^{\mathfrak{F}} f\left(x_{n, 1}^{\mathfrak{F}}\right)+\sum_{k=2}^{n} \lambda_{n, k}^{\mathfrak{F}} f\left(x_{n, k}^{\mathfrak{F}}\right),
$$


with fixed node in $x_{n, 1}^{\mathfrak{F}} \in \mathbb{R}_{\mathcal{A}_{n}}$. Further, suppose that the nodes $\left\{x_{n, k}^{\mathfrak{F}}\right\}_{k=1}^{n}$ are zeros of the 1-qORF of the first kind:

$Q_{n, \vec{a}_{2}}(x)=a_{0} \varphi_{n}(x)+a_{1} \varphi_{n-1}(x), \quad A_{n, 1}^{[Q]}:=\frac{a_{1}}{a_{0}}=-\left[\frac{\varphi_{n}(x)}{\varphi_{n-1}(x)}\right]_{x=x_{n, 1}^{\mathfrak{F}}}\left(=A_{n, 1}^{[Q]}\left(x_{n, 1}^{\mathfrak{F}}\right)\right)$,

and let the corresponding weights be given by (3.3). Then (4.3) is a rational GaussRadau quadrature rule with $\mathfrak{R}_{n}\{f\}=\mathfrak{F}\{f\}-\mathfrak{F}_{n}\{f\}=0$ for (at least) every $f \in$ $\mathcal{R}_{n, n-2}$ iff the following conditions are satisfied:

1. $A_{n, 1}^{[Q]} \in \mathbb{C}$ and $A_{n, 1}^{[Q]} \neq-E_{n} Z_{n}\left(\alpha_{n-1}\right)$;

2. $\chi_{n}^{[Q]} \in(-1, \infty)$, where $\chi_{n}^{[Q]}$ is defined as before in Lemma 4.2;

3. $\mathcal{I} m\left\{A_{n, 1}^{[Q]} \bar{E}_{n}\right\}=-\mathfrak{T}\left\{\alpha_{n}\right\}$;

4.

$$
A_{n, 1}^{[Q]} \neq-\left[\frac{\varphi_{n}(x)}{\varphi_{n-1}(x)}\right]_{x=\tilde{\alpha}} \text { for every } \tilde{\alpha} \in\left[\left(\mathcal{A}_{n-2} \cup\{\infty\}\right) \cap \overline{\mathbb{R}}_{\left\{\alpha_{n-1}, \alpha_{n}\right\}}\right] .
$$

If $\alpha_{n} \in \overline{\mathbb{R}}_{0}$, then the third condition is automatically satisfied when the second condition is satisfied. On the other hand, if $\alpha_{n} \notin \overline{\mathbb{R}}$, then the first three conditions are satisfied iff

$$
A_{n, 1}^{[Q]}=\frac{1}{\bar{E}_{n}}\left(\frac{1}{\alpha_{n-1}}-\frac{1}{\bar{\alpha}_{n}}\right) \in \mathbb{C}_{0} .
$$

Proof. First, note that $Q_{n, \vec{a}_{2}} \in{ }^{d e} \mathcal{L}_{n}$ iff the first condition is satisfied. Next, from Lemma 4.2 it follows that there exist poles $\alpha \in \overline{\mathbb{C}}_{0}$, constants $\left\{k_{n-1}, k_{n}\right\} \subset \mathbb{C}_{0}$, and HPD linear functionals $\mathfrak{G}$ so that the sequence of rational functions $\left\{\varphi_{0}, \ldots, \varphi_{n-2}\right.$, $\left.k_{n-1} \varphi_{n-1}, k_{n} \frac{Z_{\alpha}}{Z_{n}} Q_{n, \vec{a}_{2}}\right\}$, with $\frac{Z_{\alpha}}{Z_{n}} Q_{n, \vec{a}_{2}} \in{ }^{d e} \mathcal{L}_{n}^{[\alpha]}$, forms an orthonormal system in $\mathcal{L}_{n}^{[\alpha]}$ with respect to the inner product defined by $\langle f, g\rangle_{\mathfrak{G}}=\mathfrak{G}\left\{f g^{c}\right\}$ iff the second condition is satisfied. The third condition then follows from the expression for $\mathfrak{T}\{\alpha\}$ in Lemma 4.2 and the fact that the nodes of an (n)ORF are all real and distinct iff the last pole is in $\overline{\mathbb{R}}_{0}$ (i.e, iff $\mathfrak{T}\{\alpha\}=0$ ). Finally, we have that $Q_{n, \vec{a}_{2}} \in{ }^{r} \mathcal{L}_{n}$ iff the last condition is satisfied too.

Thus, the conditions given in the statement ensure the existence of the rational Gaussian quadrature formula

$$
\mathfrak{G}_{n}\{f\}:=\sum_{k=1}^{n} \lambda_{n, k}^{\mathfrak{G}} f\left(x_{n, k}^{\mathfrak{G}}\right)
$$

where $x_{n, k}^{\mathfrak{G}}=x_{n, k}^{\mathfrak{F}}$ for $k=1, \ldots, n$, and $\left(\mathfrak{G}\{f\}-\mathfrak{G}_{n}\{f\}\right)=0$ for every $f \in \mathcal{L}_{n}^{[\alpha]} \cdot \mathcal{L}_{n-1}^{c}$. Furthermore, because

$$
\mathfrak{G}\{f\}=\mathfrak{F}\{f\} \text { for every } f \in \mathcal{R}_{n-1, n-2},
$$

it follows that

$$
\mathfrak{F}\{f\}=\mathfrak{G}_{n}\{f\} \text { for every } f \in \mathcal{R}_{n-1, n-2} \supseteq \mathcal{L}_{n-1} \text {. }
$$

Due to the uniqueness of a $\left(n-1,0, \mathcal{A}_{n}, \mathfrak{F}\right)$-PRIQ with $n$ fixed nodes, it follows that

$$
\lambda_{n, k}^{\mathfrak{F}}=\lambda_{n, k}^{\mathfrak{G}} \in \mathbb{R}_{0}^{+}, \quad k=1, \ldots, n .
$$


Moreover, from the proof of Theorem 3.5 we deduce that the equality in (4.4) holds for every $f \in \mathcal{R}_{n, n-2}$ due to the fact that the nodes are zeros of a 1-qORF of the first kind.

Suppose now that $\alpha_{n} \in \overline{\mathbb{R}}_{0}$. From the second condition it then follows that

$$
\mathcal{I} m\left\{\chi_{n}^{[Q]}\right\}=0 \Leftrightarrow \mathcal{I} m\left\{a_{1} \bar{E}_{n} / a_{0}\right\} \frac{1}{\left|E_{n}\right|^{2}}\left(\frac{1}{\alpha_{n-1}}-\frac{1}{\alpha_{n}}\right)=0 .
$$

Since we assumed that $\alpha_{n} \neq \alpha_{n-1}$, this implies that

$$
\mathcal{I} m\left\{a_{1} \bar{E}_{n} / a_{0}\right\}=0=-\mathfrak{T}\left\{\alpha_{n}\right\} .
$$

On the other hand, for $\alpha_{n} \notin \overline{\mathbb{R}}$, it follows from the second and third condition that

$$
\begin{aligned}
& \left\{\begin{array}{l}
\mathcal{I} m\left\{a_{1} \bar{E}_{n} / a_{0}\right\}\left(1 / \alpha_{n-1}-\mathcal{R} e\left\{1 / \alpha_{n}\right\}\right)-\mathcal{R} e\left\{a_{1} \bar{E}_{n} / a_{0}\right\} \mathcal{I} m\left\{1 / \alpha_{n}\right\}=0 \\
\mathcal{I} m\left\{a_{1} \bar{E}_{n} / a_{0}\right\}=\mathcal{I} m\left\{1 / \alpha_{n}\right\} \neq 0
\end{array}\right. \\
& \Leftrightarrow\left\{\begin{array}{l}
\mathcal{I} m\left\{a_{1} \bar{E}_{n} / a_{0}\right\}\left(1 / \alpha_{n-1}-\mathcal{R} e\left\{1 / \alpha_{n}\right\}-\mathcal{R} e\left\{a_{1} \bar{E}_{n} / a_{0}\right\}\right)=0 \\
\mathcal{I} m\left\{a_{1} \bar{E}_{n} / a_{0}\right\}=\mathcal{I} m\left\{1 / \alpha_{n}\right\} \neq 0
\end{array}\right. \\
& \Leftrightarrow\left\{\begin{array}{l}
\mathcal{R} e\left\{a_{1} \bar{E}_{n} / a_{0}\right\}=1 / \alpha_{n-1}-\mathcal{R} e\left\{1 / \alpha_{n}\right\} \\
\mathcal{I} m\left\{a_{1} \bar{E}_{n} / a_{0}\right\}=\mathcal{I} m\left\{1 / \alpha_{n}\right\} \neq 0
\end{array} \Leftrightarrow \frac{a_{1} \bar{E}_{n}}{a_{0}}=\frac{1}{\alpha_{n-1}}-\frac{1}{\bar{\alpha}_{n}} .\right.
\end{aligned}
$$

As a result, $\chi_{n}^{[Q]}=\frac{A_{n, 1}^{[Q]}}{E_{n} Z_{n}\left(\alpha_{n-1}\right)}=\left|E_{n} Z_{n}\left(\alpha_{n-1}\right)\right|^{-2} \in \mathbb{R}_{0}^{+}$.

Finally, suppose (4.3) is a rational Gauss-Radau quadrature with $\mathfrak{R}_{n}\{f\}=0$ for (at least) every $f \in \mathcal{R}_{n, n-2}$, while the second condition is not satisfied. We then have that

$$
\lambda_{n, 1}^{\mathfrak{F}}\left|\varphi_{n-1}\left(x_{n, 1}^{\mathfrak{F}}\right)\right|^{2}+\sum_{k=2}^{n} \lambda_{n, k}^{\mathfrak{F}}\left|\varphi_{n-1}\left(x_{n, k}^{\mathfrak{F}}\right)\right|^{2}=\left|k_{n-1}\right|^{-2}>0
$$

Consider now a rational function $\varphi_{n}^{\left[\alpha_{n-1}\right]} \in{ }^{d e} \mathcal{L}_{n}$, defined by

$$
\begin{aligned}
& \varphi_{n}^{\left[\alpha_{n-1}\right]}(x)= \\
& E_{n}^{\left[\alpha_{n-1}\right]} Z_{n-1}(x)\left\{\left[1+\frac{D_{n}^{\left[\alpha_{n-1}\right]}}{Z_{n-1}(x)}\right] k_{n-1} \varphi_{n-1}(x)-\frac{1}{\bar{k}_{n-1} \bar{E}_{n-1} Z_{k-2}^{c}(x)} \varphi_{n-2}(x)\right\}
\end{aligned}
$$

where $E_{n}^{\left[\alpha_{n-1}\right]} \in \mathbb{C}_{0}$ and $\mathcal{I} m\left\{D_{n}^{\left[\alpha_{n-1}\right]}\right\}=-\frac{\mathfrak{T}\left\{\alpha_{n-2}\right\}}{\left|k_{n-1} E_{n-1}\right|^{2}}$. From Theorem 2.2 it then follows that there exist HPD linear functionals $\tilde{\mathfrak{G}}$ so that the sequence of rational functions $\left\{\varphi_{0}, \ldots, \varphi_{n-2}, k_{n-1} \varphi_{n-1}, \varphi_{n}^{\left[\alpha_{n-1}\right]}\right\}$ forms an orthonormal system in $\mathcal{L}_{n}^{\left[\alpha_{n-1}\right]}$ with respect to the inner product defined by $\langle f, g\rangle_{\tilde{\mathfrak{G}}}=\tilde{\mathfrak{G}}\left\{f g^{c}\right\}$. Moreover, the quadrature rule $(4.3)$ is then a $\left(n-1, n-1,\left\{\alpha_{1}, \ldots, \alpha_{n-1}, \alpha_{n-1}\right\}, \tilde{\mathfrak{G}}\right)$-PRIQ. Since assumption (3.2) is satisfied, it follows from Theorem 3.6 that the nodes $\left\{x_{n, k}^{\mathfrak{F}}\right\}_{k=1}^{n}$ are zeros of a 1 -qORF of the second kind of the form

$$
P_{n, \vec{b}_{2}}(x)=b_{0} \varphi_{n}^{\left[\alpha_{n-1}\right]}(x)+b_{1} \frac{Z_{n-1}(x)}{Z_{n-1}^{c}(x)} \varphi_{n-1}(x) \in{ }^{r} \mathcal{L}_{n}^{\left[\alpha_{n-1}\right]},
$$

with $\frac{Z_{n}(x)}{Z_{n-1}(x)} P_{n, \vec{b}_{2}}(x) \equiv Q_{n, \vec{a}_{2}}(x)$. In [6, Thm. 7] it has been proved then that there exists poles $\tilde{\alpha} \in \overline{\mathbb{R}}_{0}$ so that for every $k_{n} \in \mathbb{C}_{0}$, the sequence of rational functions 
$\left\{\varphi_{0}, \ldots, \varphi_{n-2}, k_{n-1} \varphi_{n-1}, k_{n} \frac{Z_{\hat{\alpha}}}{Z_{n-1}} P_{n, \vec{b}_{2}}\right\}$ forms an orthonormal system in $\mathcal{L}_{n}^{[\hat{\alpha}]}$ with respect to inner products defined by certain HPD linear functionals $\hat{\mathfrak{G}}$ on $\mathcal{L} \cdot \mathcal{L}^{c}$. But this is in contradiction with Lemma 4.2 and our assumption that the second condition is not satisfied. This concludes the proof. $\square$

4.2. Rational Gauss-Lobatto quadrature rules for $i=0$. In a similar way as above, we can now study the existence of rational Gauss-Lobatto quadrature rules for $i=0$. Since a $\left(n-1: n-2, \mathcal{A}_{n}, \mathfrak{F}\right)$-PRIQ should at least be exact for every $f \in \mathcal{R}_{n-1, n-2}$, it follows that it should also be exact for every $f \in \mathcal{R}_{n-2, n-1}$, and hence, for every $f \in \mathcal{R}_{n-2, n-2} \cdot \mathcal{S}\left\{\alpha_{n-1}\right\}$. This leaves us with the following possible situations:

1. If $\alpha_{n-1} \notin \overline{\mathbb{R}}$, it follows that $\mathcal{R}_{n-2, n-2} \cdot \mathcal{S}\left\{\alpha_{n-1}\right\}=\mathcal{R}_{n-1, n-1}\left(\supset \mathcal{R}_{n, n-2}\right)$, so that the $\left(n-1: n-2, \mathcal{A}_{n}, \mathfrak{F}\right)$-PRIQ is a $\left(n-1: n-1, \mathcal{A}_{n}, \mathfrak{F}\right)$-PRIQ (i.e., a rational Gauss-Radau quadrature rule with $i=0$ ) too.

2. If $\alpha_{n-1} \in \overline{\mathbb{R}}_{0}$, it follows that $\mathcal{R}_{n-2, n-2} \cdot \mathcal{S}\left\{\alpha_{n-1}\right\}=\mathcal{R}_{n-1, n-2}$.

For the first situation, the existence of the rational Gauss-Lobatto quadrature rule (with $i=0$ ) can easily be verified by checking the existence of the corresponding rational Gauss-Radau quadrature rule (with $i=0$ ) for the given sequence of poles $\mathcal{A}_{n}$ and fixed nodes $\mathcal{X}_{2}=\left\{x_{n, 1}^{\mathfrak{F}}, x_{n, 2}^{\mathfrak{F}}\right\}$. Thus, in what follows we can restrict ourselves to the case in which $\alpha_{n-1} \in \overline{\mathbb{R}}_{0}$. We then have the following lemma.

LEMma 4.4. A 2-qORF of the second kind

$$
P_{n, \vec{a}_{3}}(x)=a_{0} \varphi_{n}(x)+a_{1} \frac{Z_{n}(x)}{Z_{n-1}^{c}(x)} \varphi_{n-1}(x)+a_{2} \frac{Z_{n}(x)}{Z_{n-2}^{c}(x)} \varphi_{n-2}(x) \in{ }^{d e} \mathcal{L}_{n},
$$

with $\alpha_{n-1} \in \overline{\mathbb{R}}_{0}$ (hence, $Z_{n-1}^{c}(x) \equiv Z_{n-1}(x)$ ), satisfies a relation of the form

$$
P_{n, \vec{a}_{3}}(x)=E_{n}^{[P]} Z_{n}(x)\left\{\left[1+\frac{D_{n}^{[P]}}{Z_{n-1}(x)}\right] K_{n} \varphi_{n-1}(x)-\frac{C_{n}^{[P]}}{Z_{n-2}^{c}(x)} \varphi_{n-2}(x)\right\},
$$

where $K_{n} \in \mathbb{C}_{0}$,

$$
\begin{aligned}
& E_{n}^{[P]}=\frac{a_{0} E_{n}}{K_{n}} \in \mathbb{C}_{0}, \quad D_{n}^{[P]}=D_{n}+\frac{a_{1}}{a_{0} E_{n}}, \\
& C_{n}^{[P]}=\frac{\left|K_{n}\right|^{2}}{\bar{E}_{n-1}^{[P]}}\left(1-\frac{a_{2} \bar{E}_{n-1}}{a_{0} E_{n}}\right) \in \mathbb{C}, \quad \text { and } \quad E_{n-1}^{[P]}=K_{n} E_{n-1} \in \mathbb{C}_{0} .
\end{aligned}
$$

Proof. (The proof is similar to the proof of Lemma 4.1.) From Theorem 2.1 it follows that

$$
\begin{aligned}
P_{n, \vec{a}_{3}}(x)=a_{0} E_{n} Z_{n}(x)\left\{\left[1+\frac{D_{n}}{Z_{n-1}(x)}\right] \varphi_{n-1}(x)-\frac{1}{\bar{E}_{n-1} Z_{n-2}^{c}(x)} \varphi_{n-2}(x)\right\}+ \\
a_{1} \frac{Z_{n}(x)}{Z_{n-1}(x)} \varphi_{n-1}(x)+a_{2} \frac{Z_{n}(x)}{Z_{n-2}^{c}(x)} \varphi_{n-2}(x)= \\
Z_{n}(x)\left\{\left[\tilde{E}_{n}+\frac{\tilde{D}_{n}}{Z_{n-1}(x)}\right] \varphi_{n-1}(x)-\frac{\tilde{C}_{n}}{Z_{n-2}^{c}(x)} \varphi_{n-2}(x)\right\},
\end{aligned}
$$

where

$$
\tilde{E}_{n}=a_{0} E_{n}, \quad \tilde{D}_{n}=a_{0} E_{n} D_{n}+a_{1} \quad \text { and } \quad \tilde{C}_{n}=\frac{a_{0} E_{n}}{\bar{E}_{n-1}}-a_{2} .
$$


Further, for $P_{n, \vec{a}_{3}}=\frac{q_{n}}{\pi_{n}} \in{ }^{d e} \mathcal{L}_{n}$ we find with $\varphi_{k}=\frac{p_{k}}{\pi_{k}}$ that

$$
q_{n}\left(\alpha_{n-1}\right)=\tilde{E}_{n} \alpha_{n-1} p_{n-1}\left(\alpha_{n-1}\right) \neq 0,
$$

so that $\tilde{E}_{n} \neq 0$. The relation now follows by setting $\tilde{E}_{n}=E_{n}^{[P]} K_{n}$ (for an arbitrary $\left.K_{n} \in \mathbb{C}_{0}\right), \tilde{D}_{n}=D_{n}^{[P]} E_{n}^{[P]} K_{n}$, and $\tilde{C}_{n}=E_{n}^{[P]} C_{n}^{[P]}$.

As a consequence of the previous lemma and Theorem 2.2, we now can prove the following.

Lemma 4.5. Consider a 2-qORF of the second kind

$$
P_{n, \vec{a}_{3}}(x)=a_{0} \varphi_{n}(x)+a_{1} \frac{Z_{n}(x)}{Z_{n-1}^{c}(x)} \varphi_{n-1}(x)+a_{2} \frac{Z_{n}(x)}{Z_{n-2}^{c}(x)} \varphi_{n-2}(x)=\frac{q_{n}(x)}{\pi_{n}(x)} \in{ }^{d e} \mathcal{L}_{n} .
$$

Let $\alpha \in \overline{\mathbb{C}}_{0}$ be chosen in such a way that $q_{n}(\alpha) \neq 0$ and

$$
\mathfrak{T}\{\alpha\}=k \cdot\left(\mathcal{I} m\left\{\frac{a_{1} \bar{E}_{n}}{a_{0}}\right\}+\mathfrak{T}\left\{\alpha_{n}\right\}-\mathcal{R} e\left\{\frac{a_{2} \bar{E}_{n}}{a_{0} E_{n-1}}\right\} \mathfrak{T}\left\{\alpha_{n-2}\right\}\right)
$$

for some $k \in \mathbb{R}_{0}^{+}$. Then there exist HPD linear functionals $\mathfrak{G}$, for which $\left\{\varphi_{j}\right\}_{j=0}^{n-2}$ forms an orthonormal system in $\mathcal{L}_{n-2}$ and $\varphi_{n-1} \perp_{\mathfrak{G}} \mathcal{L}_{n-2}$ with respect to the inner product defined by $\langle f, g\rangle_{\mathfrak{G}}=\mathfrak{G}\left\{f g^{c}\right\}$, so that $\frac{Z_{\alpha}}{Z_{n}} P_{n, \vec{a}_{3}} \in{ }^{\text {de }} \mathcal{L}_{n}^{[\alpha]}$ and $\frac{Z_{\alpha}}{Z_{n}} P_{n, \vec{a}_{3}} \perp_{\mathfrak{G}} \mathcal{L}_{n-1}$ iff $\chi_{n}^{[P]}:=\frac{a_{2} \bar{E}_{n-1}}{a_{0} E_{n}} \in(-\infty, 1)$.

Proof. (The proof is similar to the proof of Lemma 4.2.) Since $\alpha_{n-1} \in \overline{\mathbb{R}}_{0}$, it follows from assumptions (A4)-(A5) in Theorem 2.2 that for every $K_{n} \in \mathbb{C}_{0}$, there exist HPD linear functionals $\tilde{\mathfrak{G}}$ so that $K_{n} \varphi_{n-1}$ is a nORF in ${ }^{d e} \mathcal{L}_{n-1}$ with respect to the inner product defined by $\langle f, g\rangle_{\tilde{\mathfrak{G}}}=\tilde{\mathfrak{G}}\left\{f g^{c}\right\}$. So, let $\varphi_{n}^{[\alpha]}$ be given by

$$
\begin{aligned}
\varphi_{n}^{[\alpha]}=\frac{E_{n}^{[\alpha]}}{E_{n}^{[P]}} \frac{Z_{\alpha}(x)}{Z_{n}(x)} P_{n, \vec{a}_{3}}(x) & = \\
E_{n}^{[\alpha]} Z_{\alpha}(x) & \left\{\left[1+\frac{D_{n}^{[P]}}{Z_{n-1}(x)}\right] K_{n} \varphi_{n-1}(x)-\frac{C_{n}^{[P]}}{Z_{n-2}^{c}(x)} \varphi_{n-2}(x)\right\},
\end{aligned}
$$

where $E_{n}^{[\alpha]} \in \mathbb{C}_{0}$. Then it holds that $\varphi_{n}^{[\alpha]}$ satisfies assumptions (A1)-(A4) in Theorem 2.2. From assumption (A6) it now follows that $C_{n}^{[P]}$ should be equal to $\left(\bar{K}_{n} \bar{E}_{n-1}\right)^{-1}$. From the previous lemma we deduce that this can only be for $\left|K_{n}\right|^{-2}=1-\chi_{n}^{[P]}$, which implies that $\chi_{n}^{[P]}$ should be in $(-\infty, 1)$. Finally, we have that

$$
\begin{aligned}
& \operatorname{Im} m\left\{D_{n}^{[P]}\right\}= \mathcal{I} m\left\{D_{n}\right\}+\mathcal{I} m\left\{\frac{a_{1}}{a_{0} E_{n}}\right\}=\operatorname{Im}\left\{\frac{a_{1}}{a_{0} E_{n}}\right\}+\frac{\mathfrak{T}\left\{\alpha_{n}\right\}}{\left|E_{n}\right|^{2}}-\frac{\mathfrak{T}\left\{\alpha_{n-2}\right\}}{\left|E_{n-1}\right|^{2}}= \\
& \frac{\mathcal{I} m\left\{\frac{a_{1} \bar{E}_{n}}{a_{0}}\right\}+\mathfrak{T}\left\{\alpha_{n}\right\}}{\left|E_{n}\right|^{2}}-\chi_{n}^{[P]} \frac{\mathfrak{T}\left\{\alpha_{n-2}\right\}}{\left|E_{n-1}\right|^{2}}-\frac{\mathfrak{T}\left\{\alpha_{n-2}\right\}}{\left|E_{n-1}^{[P]}\right|^{2}}= \\
& \frac{\mathcal{I} m\left\{\frac{a_{1} \bar{E}_{n}}{a_{0}}\right\}+\mathfrak{T}\left\{\alpha_{n}\right\}-\left|\frac{E_{n}}{E_{n-1}}\right|^{2} \chi_{n}^{[P]} \mathfrak{T}\left\{\alpha_{n-2}\right\}}{\left|E_{n}\right|^{2}}-\frac{\mathfrak{T}\left\{\alpha_{n-2}\right\}}{\left|E_{n-1}^{[P]}\right|^{2}}=\frac{\mathfrak{T}\{\alpha\}}{k\left|E_{n}\right|^{2}}-\frac{\mathfrak{T}\left\{\alpha_{n-2}\right\}}{\left|E_{n-1}^{[P]}\right|^{2}},
\end{aligned}
$$


where the second equality follows from assumption (A5) applied on $D_{n}$, and the last equality is due to

$$
\left|\frac{E_{n}}{E_{n-1}}\right|^{2} \chi_{n}^{[P]}=\frac{a_{2} \bar{E}_{n}}{a_{0} E_{n-1}} \in \mathbb{R}
$$

Consequently, assumption (A5) in Theorem 2.2 is satisfied too for every $E_{n}^{[\alpha]} \in \mathbb{C}_{0}$ if $\mathfrak{T}\{\alpha\}=0$, respectively for $\left|E_{n}^{[\alpha]}\right|^{2}=k\left|E_{n}\right|^{2} \in \mathbb{R}_{0}^{+}$if $\mathfrak{T}\{\alpha\} \neq 0$.

Before we can prove our second main result, we need the following lemma.

LEMmA 4.6. Suppose the nORFs $\varphi_{k} \in{ }^{d e} \mathcal{L}_{k}, k=1, \ldots, n$. Then the nORFs $\varphi_{n}$ and $\varphi_{n-1}$ have no common zeros.

Proof. Set $\varphi_{k}=\frac{p_{k}}{\pi_{k}}$. From Theorem 2.1 it then follows that there exist polynomials $q_{1}^{(k)} \in \mathcal{P}_{1}$ and constants $q_{0}^{(k)} \in \mathbb{C}_{0}$ so that

$$
p_{k}(x)=q_{1}^{(k)}(x) p_{k-1}(x)+q_{0}^{(k)}\left(1-x / \alpha_{k-1}\right)\left(1-x / \bar{\alpha}_{k-2}\right) p_{k-2}(x), \quad k=2, \ldots, n .
$$

So, let $k=n$ and suppose that $p_{k-1}(\alpha)=p_{k}(\alpha)=0$ for $\alpha \in \overline{\mathbb{C}}$ (recall that ' $p_{k}(\infty)=0$ ' means that ' $p_{k} \in \mathcal{P}_{k-1}$ '). Since $\varphi_{k} \in{ }^{e} \mathcal{L}_{k}$ and $\varphi_{k-1} \in{ }^{d} \mathcal{L}_{k-1}$, it follows that $\alpha \neq \alpha_{k-1}$ and $\alpha \neq \bar{\alpha}_{k-2}$, and hence, that $p_{k-2}(\alpha)=p_{k-1}(\alpha)=0$. Thus, continuing for $k=$ $n-1, \ldots, 2$, we find that $\alpha \notin\left\{\alpha_{k-1}, \bar{\alpha}_{k-2}\right\}_{k=2}^{n}$, and that $p_{0}(\alpha)=p_{1}(\alpha)=0$. This, however, contradicts the fact that $\varphi_{0} \equiv p_{0} \in \mathbb{C}_{0}$.

Finally, we are able to prove the following.

THEOREM 4.7. Consider a quadrature formula of the form

$$
\mathfrak{F}_{n}\{f\}=\lambda_{n, 1}^{\mathfrak{F}} f\left(x_{n, 1}^{\mathfrak{F}}\right)+\lambda_{n, 2}^{\mathfrak{F}} f\left(x_{n, 2}^{\mathfrak{F}}\right)+\sum_{k=3}^{n} \lambda_{n, k}^{\mathfrak{F}} f\left(x_{n, k}^{\mathfrak{F}}\right),
$$

with fixed distinct nodes in $\left\{x_{n, 1}^{\mathfrak{F}}, x_{n, 2}^{\mathfrak{F}}\right\} \in \mathbb{R}_{\mathcal{A}_{n}}$. Further, let the functions $a(\tilde{x})$ and $b(\tilde{x})$ be defined by

$$
a(\tilde{x})=\left[\frac{Z_{n-1}(x)}{Z_{n}(x)}\right]_{x=\tilde{x}} \cdot\left[\frac{\varphi_{n}(x)}{\varphi_{n-1}(x)}\right]_{x=\tilde{x}} \quad \text { and } \quad b(\tilde{x})=\left[\frac{Z_{n-1}(x)}{Z_{n-2}^{c}(x)}\right]_{x=\tilde{x}} \cdot\left[\frac{\varphi_{n-2}(x)}{\varphi_{n-1}(x)}\right]_{x=\tilde{x}} .
$$

Suppose the nodes $\left\{x_{n, k}^{\mathfrak{F}}\right\}_{k=1}^{n}$ are zeros of the $2-q O R F$ of the second kind:

$P_{n, \vec{a}_{3}}(x)=a_{0} \varphi_{n}(x)+a_{1} \frac{Z_{n}(x)}{Z_{n-1}(x)} \varphi_{n-1}(x)+a_{2} \frac{Z_{n}(x)}{Z_{n-2}^{c}(x)} \varphi_{n-2}(x) \quad\left(Z_{n-1}(x) \equiv Z_{n-1}^{c}(x)\right)$,

with

$$
\begin{array}{r}
A_{n, 1}^{[P]}:=\frac{a_{1}}{a_{0}}=\frac{a\left(x_{n, 1}^{\mathfrak{F}}\right) b\left(x_{n, 2}^{\mathfrak{F}}\right)-a\left(x_{n, 2}^{\mathfrak{F}}\right) b\left(x_{n, 1}^{\mathfrak{F}}\right)}{b\left(x_{n, 1}^{\mathfrak{F}}\right)-b\left(x_{n, 2}^{\mathfrak{F}}\right)}\left(=A_{n, 1}^{[P]}\left(x_{n, 1}^{\mathfrak{F}}, x_{n, 2}^{\mathfrak{F}}\right)\right) \quad \text { and } \\
A_{n, 2}^{[P]}:=\frac{a_{2}}{a_{0}}=\frac{a\left(x_{n, 2}^{\mathfrak{F}}\right)-a\left(x_{n, 1}^{\mathfrak{F}}\right)}{b\left(x_{n, 1}^{\mathfrak{F}}\right)-b\left(x_{n, 2}^{\mathfrak{F}}\right)}\left(=A_{n, 2}^{[P]}\left(x_{n, 1}^{\mathfrak{F}}, x_{n, 2}^{\mathfrak{F}}\right)\right)
\end{array}
$$

and let the corresponding weights be given by (3.5). Then (4.6) is a rational GaussLobatto quadrature rule with $\mathfrak{R}_{n}\{f\}=\mathfrak{F}\{f\}-\mathfrak{F}_{n}\{f\}=0$ for (at least) every $f \in$ $\mathcal{R}_{n-1, n-2}$ iff the following conditions are satisfied: 
1. $\left\{b\left(x_{n, 1}^{\mathfrak{F}}\right), b\left(x_{n, 2}^{\mathfrak{F}}\right)\right\} \subset \mathbb{C}$, and $b\left(x_{n, 1}^{\mathfrak{F}}\right)-b\left(x_{n, 2}^{\mathfrak{F}}\right) \neq 0$;

2.

$$
\frac{\bar{E}_{n-1}\left[Z_{n-1}\left(\alpha_{n}\right)+D_{n}+A_{n, 1}^{[P]} / E_{n}\right]}{1-\chi_{n}^{[P]}} \neq b\left(\alpha_{n}\right) \quad \text { if } \alpha_{n} \neq \alpha_{n-1} \text { and } \alpha_{n} \in \overline{\mathbb{R}}_{0}
$$

where $\chi_{n}^{[P]}$ is defined as before in Lemma 4.5;

3. $\chi_{n}^{[P]} \in(-\infty, 1)$;

4. $\mathcal{I} m\left\{A_{n, 1}^{[P]} \bar{E}_{n}\right\}-A_{n, 2}^{[P]} \frac{\bar{E}_{n}}{E_{n-1}} \mathfrak{T}\left\{\alpha_{n-2}\right\}=-\mathfrak{T}\left\{\alpha_{n}\right\}$;

5. $a(\tilde{\alpha})+A_{n, 1}^{[P]}+A_{n, 2}^{[P]} b(\tilde{\alpha}) \neq 0$ for every $\tilde{\alpha} \in\left[\left(\mathcal{A}_{n-2} \cup\{\infty\}\right) \cap \overline{\mathbb{R}}_{\left\{\alpha_{n-1}, \alpha_{n}\right\}}\right]$.

Proof. First, note that $P_{n, \vec{a}_{3}} \notin{ }^{d e} \mathcal{L}_{n}$ if $a_{0}=0$. Hence, we may assume that $a_{0} \neq 0$ so that the coefficients $A_{n, 1}^{[P]}$ and $A_{n, 2}^{[P]}$ are well defined. We then have that $x_{n, j}^{\mathfrak{F}}$, $j=1,2$, are zeros of $P_{n, \vec{a}_{3}}$ iff

$$
\varphi_{n}\left(x_{n, j}^{\mathfrak{F}}\right)+A_{n, 1}^{[P]} \frac{Z_{n}\left(x_{n, j}^{\mathfrak{F}}\right)}{Z_{n-1}\left(x_{n, j}^{\mathfrak{F}}\right)} \varphi_{n-1}\left(x_{n, j}^{\mathfrak{F}}\right)+A_{n, 2}^{[P]} \frac{Z_{n}\left(x_{n, j}^{\mathfrak{F}}\right)}{Z_{n-2}^{c}\left(x_{n, j}^{\mathfrak{F}}\right)} \varphi_{n-2}\left(x_{n, j}^{\mathfrak{F}}\right)=0, \quad j=1,2 .
$$

Suppose now that the $j$ th node is a zero of $\varphi_{n-1}$. From (4.5) it then follows that

$$
0=C_{n}^{[P]} \frac{Z_{n}\left(x_{n, j}^{\mathfrak{F}}\right)}{Z_{n-2}^{c}\left(x_{n, j}^{\mathfrak{F}}\right)} \varphi_{n-2}\left(x_{n, j}^{\mathfrak{F}}\right)
$$

Due to the previous lemma, a zero of $\varphi_{n-1}$ cannot be a zero of $\varphi_{n-2}$, so that $C_{n}^{[P]}=0$. As a result, the third condition is not satisfied. For this reason, the first part of the first condition ensures that none of the nodes are zeros of $\varphi_{n-1}$, and that $\chi_{n}^{[P]} \neq 1$. The expressions for $A_{n, 1}^{[P]}$ and $A_{n, 2}^{[P]}$ then follow by dividing the left-hand side in (4.7) with $\frac{Z_{n}\left(x_{n, j}^{\mathfrak{F}}\right)}{Z_{n-1}\left(x_{n, j}^{\mathfrak{F}}\right)} \varphi_{n-1}\left(x_{n, j}^{\mathfrak{F}}\right)$, and solving for $A_{n, 1}^{[P]}$ and $A_{n, 2}^{[P]}$. Further, for $b\left(x_{n, 1}^{\mathfrak{F}}\right)=b\left(x_{n, 2}^{\mathfrak{F}}\right)$ it holds that

$$
a\left(x_{n, 1}^{\mathfrak{F}}\right)-a\left(x_{n, 2}^{\mathfrak{F}}\right)=E_{n}\left[Z_{n-1}\left(x_{n, 1}^{\mathfrak{F}}\right)-Z_{n-1}\left(x_{n, 2}^{\mathfrak{F}}\right)\right] \neq 0
$$

so that $a_{0} \neq 0$ iff the second part of the first condition is satisfied.

Next, for $\alpha_{n} \neq \alpha_{n-1}$ we deduce from (4.5) that $P_{n, \vec{a}_{3}} \in \mathcal{L}_{n} \backslash \mathcal{L}_{n-1}$ (and hence, in ${ }^{d e} \mathcal{L}_{n}$ due to $\left.a_{0} \neq 0\right)$ iff the second condition is satisfied. Note that $b\left(\alpha_{n}\right)$ is well defined. Indeed, since $\varphi_{n-1} \in{ }^{d e} \mathcal{L}_{n-1}$, it follows that $\left[\frac{\varphi_{n-2}(x)}{\varphi_{n-1}(x)}\right]_{x=\alpha_{n}}$ is finite whenever $\alpha_{n}=\bar{\alpha}_{n-2}$. Similar as in the proof of Theorem 4.3, we then have that

1. conditions 3-5 ensure the existence of a rational Gaussian quadrature formula $\mathfrak{G}_{n}\{f\}$ so that $\mathfrak{F}\{f\}=\mathfrak{G}\{f\}=\mathfrak{G}_{n}\{f\}$ for every $f \in \mathcal{R}_{n-1, n-2}$, and hence, $x_{n, k}^{\mathfrak{F}}=x_{n, k}^{\mathfrak{G}}$ and $\lambda_{n, k}^{\mathfrak{F}}=\lambda_{n, k}^{\mathfrak{G}} \in \mathbb{R}_{0}^{+}$for $k=1, \ldots, n$;

2. supposing the rational Gauss-Lobatto quadrature exists, while the fixed nodes are zeros of a 2 -qORF of the second kind that does not satisfy the third condition, leads to a contradiction.

Finally, note that we only need to verify the second condition if $\alpha_{n} \in \overline{\mathbb{R}}_{0}$, because conditions 3 and 4 ensure that all the zeros are real. This ends the proof. 
4.3. Construction of rational Gauss-type quadrature rules. In the previous subsections, we related the rational Gauss-Radau and Gauss-Lobatto quadrature rules (for respectively $i=1$ and $i=0$ ) with certain rational Gaussian quadrature rules by means of the Lemmas 4.2 and 4.5 respectively. Supposing the quadrature rule exists for a given (set of) node(s), the aim of this subsection is then to investigate the construction of the quadrature rule (i.e., the computation of the nodes and weights in the quadrature formula). For this, let $X$ be fixed to either $Q$ or $P$, and suppose that the matrices $\mathbf{J}_{n}, \mathbf{D}_{n}, \mathbf{I}_{n}$ and $\mathbf{S}_{n}$, are defined as above in Theorem 2.3. Further, let $\chi_{n}^{[Q]}, \chi_{n}^{[P]}, A_{n, 1}^{[Q]}$ and $A_{n, 1}^{[P]}$, be defined as before in Lemmas 4.2 and 4.5, and Theorems 4.3 and 4.7 , and set

$$
c_{n-2}^{[X]}=\frac{c_{n-2}^{[\varphi]}}{\eta K_{n}^{[X]}}, \quad a_{n-1}^{[X]}=\bar{c}_{n-2}^{[X]}, \quad \text { and } \quad b_{n-1}^{[X]}=\left(b_{n-1}^{[\varphi]}-A_{n, 1}^{[X]} c_{n-1}^{[\varphi]}\right) / L_{n}^{[X]},
$$

where $|\eta|=1$,

$$
K_{n}^{[X]}=\left\{\begin{array}{ll}
\sqrt{1+\chi_{n}^{[Q]}}, & X=Q \\
1 / \sqrt{1-\chi_{n}^{[P]}}, & X=P
\end{array} \quad \text { and } \quad L_{n}^{[X]}= \begin{cases}1+\chi_{n}^{[Q]}, & X=Q \\
1, & X=P .\end{cases}\right.
$$

With this we can define the modified matrices

$$
\mathbf{J}_{n}^{[X]}=\left(\begin{array}{ccc}
\mathbf{J}_{n-1} & \overrightarrow{0}_{n-2} \\
\overrightarrow{0}_{n-2}^{T} & a_{n-1}^{[X]} & c_{n-2}^{[X]} \\
b_{n-1}^{[X]}
\end{array}\right), \mathbf{S}_{n}^{[X]}=\left(\begin{array}{ccc} 
& \mathbf{S}_{n-1} & \overrightarrow{0}_{n-2} \\
\overrightarrow{0}_{n-2}^{T} & \frac{a_{n-1}^{[X]}}{Z_{n-2}^{c}\left(\alpha_{n-2}\right)} & 0
\end{array}\right),
$$

and

$$
\mathbf{B}_{n}^{[X]}=\mathbf{J}_{n}^{[X]} \mathbf{D}_{n}+\mathbf{I}_{n}-\mathbf{S}_{n}^{[X]}, \quad \text { where } \overrightarrow{0}_{n-2}=\left(\begin{array}{lll}
0 & \ldots & 0
\end{array}\right)^{T} \in \mathbb{C}^{n-2},
$$

and modified column vector

$$
\vec{\varphi}_{n}^{[X]}(x)=\left(\begin{array}{llll}
\varphi_{0}(x) & \varphi_{1}(x) & \ldots & K_{n}^{[X]} \varphi_{n-1}(x)
\end{array}\right)^{T} .
$$

We then have the following theorem for the construction of the rational Gauss-Radau and Gauss-Lobatto quadrature rules from the previous subsections.

TheOREM 4.8. Given the notation introduced above, the nodes $\left\{x_{n, k}^{\mathfrak{F}}\right\}$ in the rational Gauss-Radau quadrature rule (4.3) $(X=Q)$, respectively in the rational Gauss-Lobatto quadrature rule (4.6) $(X=P)$, are eigenvalues of the GEP

$$
\mathbf{J}_{n}^{[X]} \vec{v}_{n, k}^{[X]}=x_{n, k}^{\mathfrak{F}} \mathbf{B}_{n}^{[X]} \vec{v}_{n, k}^{[X]},
$$

with

$\vec{v}_{n, k}^{[X]}=\eta_{n}^{[X]}\left\{\sum_{j=0}^{n-2}\left|\varphi_{j}\left(x_{n, k}^{\mathfrak{F}}\right)\right|^{2}+\left[K_{n}^{[X]}\right]^{2} \cdot\left|\varphi_{n-1}\left(x_{n, k}^{\mathfrak{F}}\right)\right|^{2}\right\}^{-1 / 2} \quad \vec{\varphi}_{n}^{[X]}\left(x_{n, k}^{\mathfrak{F}}\right), \quad\left|\eta_{n}^{[X]}\right|=1$,

the corresponding normalized eigenvector. The corresponding weights satisfy

$$
\lambda_{n, k}^{\mathfrak{F}}=\left|v_{n, k}^{[X]}\right|^{2}\|1\|_{\mathfrak{F}}^{2},
$$

where $v_{n, k}^{[X]}$ denotes the first entry in $\vec{v}_{n, k}^{[X]}$.

Proof. The statement directly follows from Lemmas 4.1 and 4.2 for $X=Q$ (respectively from Lemmas 4.4 and 4.5 for $X=P$ ), together with (4.1) and Theorem 2.1. 
5. Numerical examples. In the numerical examples that follow, we construct rational Gauss-type quadrature formulas for the approximation of

$$
\mathfrak{F}\{f\}=J_{w}(f):=\int_{I} f(x) w(x) d x,
$$

where $I=[-1,1]$ and $w(x)$ is the Chebyshev weight function of the first kind $w(x)=$ $1 / \sqrt{1-x^{2}}$.

Let $x=J(z)$ denote the Joukowski Transformation $x=\frac{1}{2}\left(z+z^{-1}\right)$, which maps the open unit disc $\mathbb{D}=\{z \in \mathbb{C}:|z|<1\}$ onto the cut Riemann sphere $\overline{\mathbb{C}}_{I}$ and the unit circle $\mathbb{T}=\{z \in \mathbb{C}:|z|=1\}$ onto the interval $I$. The inverse mapping is denoted by $z=J^{\text {inv }}(x)$ and is chosen so that $z \in \mathbb{D}$ if $x \in \overline{\mathbb{C}}_{I}$. Further, with the sequence $\mathcal{A}=\left\{\alpha_{1}, \alpha_{2}, \ldots\right\} \subset \overline{\mathbb{C}}_{I}$ we associate a sequence $\mathcal{B}=\left\{\beta_{1}, \beta_{2}, \ldots\right\} \subset \mathbb{D}$, so that $\beta_{k}=J^{i n v}\left(\alpha_{k}\right)$. The so-called Chebyshev nORFs (with respect to the Chebyshev weight function $w(x)$ and inner product $\left.\langle f, g\rangle_{w}=J_{w}\left(f g^{c}\right)\right)$, with arbitrary complex poles outside $I$, are then given by (see [8])

$$
\varphi_{0}(x) \equiv \frac{1}{\sqrt{\pi}}, \quad \varphi_{k}(x)=\sqrt{\frac{1-\left|\beta_{k}\right|^{2}}{2 \pi}}\left(\frac{z B_{k-1}^{c}(z)}{1-\beta_{k} z}+\frac{1}{\left(z-\beta_{k}\right) B_{k-1}(z)}\right), k \geqslant 1,
$$

where

$$
B_{0}(z) \equiv 1, \quad B_{k}(z)=\prod_{j=1}^{k} \frac{z-\beta_{j}}{1-\bar{\beta}_{j} z}, \quad k \geqslant 1 .
$$

These nORFs are regular and satisfy the three-term recurrence relation (2.4) with coefficients (see [16, Sect. 4] and [19, Thm. 3.5])

$$
E_{k}=\frac{2 \sqrt{\left(1-\left|\beta_{k}\right|^{2}\right)\left(1-\left|\beta_{k-1}\right|^{2}\right)}\left(1-\beta_{k} \beta_{k-1}\right)}{\left(1+\beta_{k}^{2}\right)\left(1+\beta_{k-1}^{2}\right)}, k>1,
$$

and

$$
\begin{aligned}
& D_{k}=-\frac{1+\beta_{k-1}^{2}}{2\left(1-\left|\beta_{k-1}\right|^{2}\right)} \times \\
& \quad \frac{\left(1-\left|\beta_{k-1}\right|^{2}\right)\left(\beta_{k}+\bar{\beta}_{k-2}\right)+2 \mathcal{R} e\left\{\beta_{k-1}\right\}\left(1-\beta_{k} \bar{\beta}_{k-2}\right)}{\left(1-\beta_{k} \beta_{k-1}\right)\left(1-\beta_{k-1} \bar{\beta}_{k-2}\right)}, k>1,
\end{aligned}
$$

were we put by convention $\beta_{0}=J^{i n v}\left(\alpha_{0}\right)=0$. For $k=1$, the coefficients are given by

$$
E_{1}=\frac{\sqrt{2\left(1-\left|\beta_{1}\right|^{2}\right)}}{1+\beta_{1}^{2}} \text { and } D_{1}=-\beta_{1} .
$$

Unless stated differently, the computations in the examples were done in double precision using MATLAB ${ }^{\circledR} 7 .{ }^{4}$

${ }^{4}$ MATLAB is a registered trademark of The MathWorks, Inc. 
EXAMPLE 5.1. For the first example, we consider the sequence of poles $\mathcal{A}_{1}=$ $\{-\alpha, \alpha,-\alpha, \alpha, \ldots\} \subset \mathbb{R}_{I}$, with $\alpha>1$. We then numerically approximate

$$
J_{w}\left(\varphi_{k} \varphi_{l}^{c}\right)=\delta_{k, l}, \quad n-2 \leqslant k \leqslant n, \quad n-2 \leqslant l<n,
$$

where the $\varphi_{k}$ 's are the Chebyshev nORFs (5.1) with poles in $\mathcal{A}_{1}$, by means of an $n$th rational Gauss-Radau quadrature formula

$$
J_{n}\left(\varphi_{k} \varphi_{l}^{c}\right)=\lambda_{n, 1} \varphi_{k}\left(x_{n, 1}\right) \varphi_{l}^{c}\left(x_{n, 1}\right)+\sum_{j=2}^{n} \lambda_{n, j} \varphi_{k}\left(x_{n, j}\right) \varphi_{l}^{c}\left(x_{n, j}\right)
$$

with fixed node in $x_{n, 1}=1$ or $x_{n, 1}=-1$. Thus, for $n>1$ we have with $\beta=J^{\text {inv }}(\alpha) \in$ $(0,1)$ that

$$
A_{n, 1}^{[Q]}(-1)=-1 / A_{n, 1}^{[Q]}(1)= \begin{cases}\frac{1+\beta}{1-\beta}, & n=\text { odd } \\ \frac{1-\beta}{1+\beta}, & n=\text { even } .\end{cases}
$$

Further, the coefficients (2.5) are given by

$$
c_{0}^{[\varphi]}=a_{1}^{[\varphi]}=\frac{1+\beta^{2}}{\sqrt{2\left(1-\beta^{2}\right)}}, \quad c_{k-1}^{[\varphi]}=a_{k}^{[\varphi]}=\frac{1+\beta^{2}}{2\left(1-\beta^{2}\right)}, \quad k>1,
$$

and

$$
b_{0}^{[\varphi]}=-\beta, \quad b_{1}^{[\varphi]}=-\frac{\beta\left(1+\beta^{2}\right)}{2\left(1-\beta^{2}\right)}, \quad b_{k}^{[\varphi]}=0, \quad k>1 .
$$

Thus, we have that $A_{n, 1}^{[Q]}( \pm 1) \in \mathbb{C}$, while

$$
\chi_{n}^{[Q]}(1)=\left\{\begin{array}{ll}
-\frac{2 \beta}{(1+\beta)^{2}}, & n=\text { odd } \\
\frac{2 \beta}{(1-\beta)^{2}}, & n=\text { even }
\end{array} \quad \text { and } \quad \chi_{n}^{[Q]}(-1)= \begin{cases}\frac{2 \beta}{(1-\beta)^{2}}, & n=\text { odd } \\
-\frac{2 \beta}{(1+\beta)^{2}}, & n=\text { even },\end{cases}\right.
$$

so that the four conditions in Theorem 4.3 are all satisfied. Hence, these rational Gauss-Radau quadrature rules exist, and we can use Theorem 4.8 to compute their nodes and weights. Tables 5.1 and 5.2 then show the absolute error of the approximation for several values of $n$ and $\alpha$. To get an idea of the accuracy of the computed nodes and weights, we included the absolute error $\epsilon_{n}\left(\left|\varphi_{0}\right|^{2}\right)$ of the approximation of $J_{w}\left(\left|\varphi_{0}\right|^{2}\right)=1$ too, as well as the difference in absolute value between the fixed node $x_{n, 1}$ and the nearest computed node $x_{n, j}^{[\text {comp }]}$. The numerical results clearly show that the quadrature rules are exact for every $f \in \mathcal{R}_{n, n-2}$, but not for $f \in \mathcal{R}_{n-1, n-1} \backslash \mathcal{R}_{n-1, n-2}$.

EXAMPLE 5.2. For the second and last example, we consider the function

$$
f(x)=\frac{1}{\pi} \sin \left\{\frac{1}{\left(x^{2}-J(\beta)^{2}\right)\left(x^{2}-J(\mathbf{i} \beta)^{2}\right)}\right\}, \quad \beta \in(0,1),
$$

which is analogous to the one in Example 5.4 from [19]. This function has essential singularities in $x=J\left(\mathbf{i}^{j} \beta\right), j=0, \ldots, 3$. For $\beta$ very close to one, this function is extremely oscillating near the center and near the endpoints of the interval $I$. Since an essential singularity can be viewed as a pole of infinity multiplicity, this suggests taking

$$
\mathcal{A}_{2}=\left\{\alpha_{k}=J\left(\mathbf{i}^{k-1} \beta\right), k=1,2, \ldots\right\}
$$


TABLE 5.1

Absoloute error in the rational Gauss-Radau quadrature formulas with fixed node in 1 for the estimation of $J_{w}\left(\varphi_{k} \varphi_{l}^{c}\right)$.

\begin{tabular}{|c|c|c|c|c|c|}
\hline$\alpha$ & $n$ & $(k, l)$ & error & $\epsilon_{n}\left(\left|\varphi_{0}\right|^{2}\right)$ & $\min _{1 \leqslant j \leqslant n}\left\{x_{n, j}^{[c o m p]}-1\right.$ \\
\hline \multirow[t]{3}{*}{2} & 8 & $\begin{array}{l}(6,6) \\
(7,6) \\
(8,6) \\
(7,7)\end{array}$ & $\begin{array}{l}3.1107 e-015 \\
6.7671 e-016 \\
3.7793 e-015 \\
5.0000 e-001\end{array}$ & $1.1102 e-015$ & $4.4409 e-016$ \\
\hline & 32 & $\begin{array}{l}(30,30) \\
(31,30) \\
(32,30) \\
(31,31)\end{array}$ & $\begin{array}{l}6.5060 e-014 \\
3.4982 e-014 \\
6.9833 e-014 \\
5.0000 e-001\end{array}$ & $2.2204 e-016$ & $6.6613 e-016$ \\
\hline & 128 & $\begin{array}{l}(126,126) \\
(127,126) \\
(128,126) \\
(127,127)\end{array}$ & $\begin{array}{l}8.6710 e-014 \\
2.4629 e-013 \\
1.6776 e-013 \\
5.0000 e-001\end{array}$ & $8.6597 e-015$ & $2.2204 e-016$ \\
\hline \multirow[t]{3}{*}{1.1} & 8 & $\begin{array}{l}(6,6) \\
(7,6) \\
(8,6) \\
(7,7)\end{array}$ & $\begin{array}{l}2.8869 e-015 \\
5.9539 e-015 \\
2.9089 e-014 \\
9.0909 e-001\end{array}$ & $6.6613 e-016$ & $3.3307 e-016$ \\
\hline & 32 & $\begin{array}{l}(30,30) \\
(31,30) \\
(32,30) \\
(31,31)\end{array}$ & $\begin{array}{l}2.5824 e-013 \\
4.6078 e-014 \\
3.0015 e-013 \\
9.0909 e-001\end{array}$ & $5.3291 e-015$ & $4.4409 e-016$ \\
\hline & 128 & $\begin{array}{l}(126,126) \\
(127,126) \\
(128,126) \\
(127,127) \\
\end{array}$ & $\begin{array}{l}5.8576 e-013 \\
5.0504 e-016 \\
7.8601 e-013 \\
9.0909 e-001\end{array}$ & $6.2728 e-014$ & $4.4409 e-016$ \\
\hline \multirow[t]{3}{*}{1.001} & 8 & $\begin{array}{l}(6,6) \\
(7,6) \\
(8,6) \\
(7,7)\end{array}$ & $\begin{array}{l}2.6725 e-012 \\
6.9373 e-014 \\
3.7845 e-012 \\
9.9900 e-001\end{array}$ & $8.9040 e-014$ & $8.8818 e-016$ \\
\hline & 32 & $\begin{array}{l}(30,30) \\
(31,30) \\
(32,30) \\
(31,31)\end{array}$ & $\begin{array}{l}1.9464 e-011 \\
3.3857 e-013 \\
2.2508 e-011 \\
9.9900 e-001\end{array}$ & $1.8097 e-013$ & $4.4409 e-016$ \\
\hline & 128 & $\begin{array}{l}(126,126) \\
(127,126) \\
(128,126) \\
(127,127)\end{array}$ & $\begin{array}{l}5.3282 e-010 \\
1.1467 e-011 \\
5.2787 e-010 \\
9.9900 e-001\end{array}$ & $5.8442 e-013$ & $2.2204 e-015$ \\
\hline
\end{tabular}

We then numerical approximate $J_{w}(f)$ by means of an nth rational Gauss-type (Gaussian, Gauss-Radau and/or Gauss-Lobatto) quadrature rule $J_{n}(f)$.

Note that the rational Gaussian quadrature rule only exists for $n$ odd, while the rational Gauss-Radau and Gauss-Lobatto quadrature rules can only exist for $n$ even. 
TABle 5.2

Absoloute error in the rational Gauss-Radau quadrature formulas with fixed node in -1 for the estimation of $J_{w}\left(\varphi_{k} \varphi_{l}^{c}\right)$.

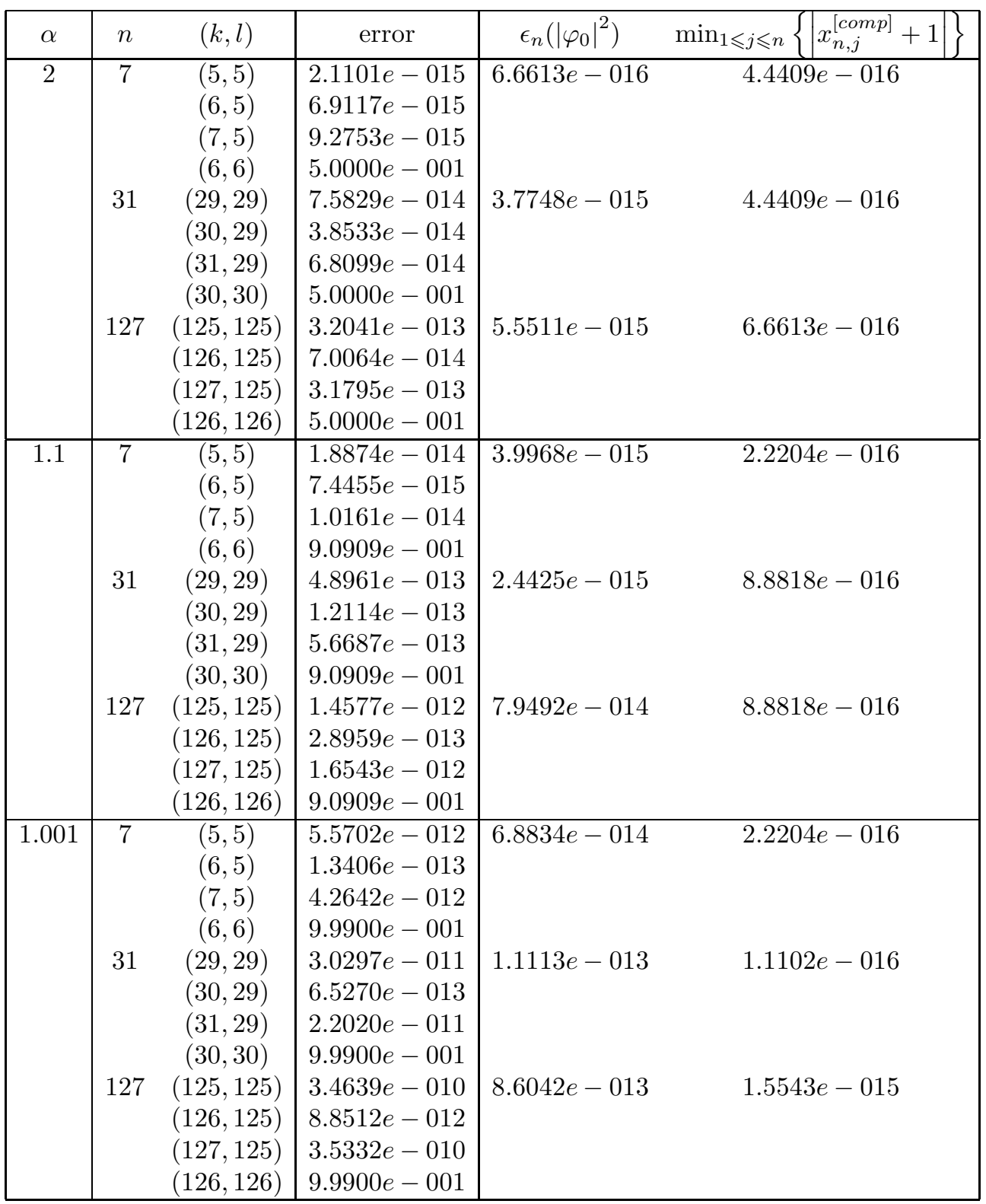

The coefficients (2.5) are now given by

$$
c_{k-1}^{[\varphi]}=\bar{a}_{k}^{[\varphi]}= \begin{cases}\frac{1+\beta^{2}}{\sqrt{2\left(1-\beta^{2}\right)}}, & k=1 \\ \frac{1+\beta^{2}}{2\left(1-(-1)^{k} \mathbf{i} \beta^{2}\right)}, & k>1 \\ 24 & \end{cases}
$$


and

$$
b_{k-1}^{[\varphi]}=\left\{\begin{array}{ll}
\beta, & k=1 \\
\frac{\beta\left(1+\beta^{2}\right)\left[2+\mathbf{i}\left(1-\beta^{2}\right)\right]}{2\left(1-\beta^{2}\right)\left(1-\mathbf{i} \beta^{2}\right)}, & k=2 \\
0, & k=2 m+1 \\
(-1)^{m} \frac{\left.\beta\left(1+\beta^{2}\right)\left[\left(1+\beta^{2}\right)+\mathbf{i}\left(1-\beta^{2}\right)\right)\right]}{\left(1-\beta^{2}\right)\left(1-\mathbf{i} \beta^{2}\right)^{2}}, & k=2 m+2,
\end{array} \quad m=1,2, \ldots .\right.
$$

Further, since the last pole is complex for $n$ even, the nth rational Gauss-Radau quadrature rule is unique (it is the nth rational Gaussian quadrature rule with the last two poles interchanged), so that (see Theorem 4.3),

$$
A_{2 m, 1}^{[Q]}=-(-1)^{m} \frac{\beta\left[\left(1-\beta^{2}\right)+\mathbf{i}\left(1+\beta^{2}\right)\right]}{\left(1+\mathbf{i} \beta^{2}\right)\left(1-\beta^{2}\right)} \quad \text { and } \quad \chi_{2 m}^{[Q]}=\frac{2 \beta^{2}}{\left(1-\beta^{2}\right)^{2}}, \quad m=1,2, \ldots .
$$

For the nth rational Gauss-Lobatto quadrature rule, with $n=2 m \geqslant 4$, we consider the case of fixed nodes in $x_{n, 1}=-x_{n, 2}=1$. We then have that

$$
A_{2 m, 1}^{[P]}(1,-1)=0, \quad A_{2 m, 2}^{[P]}(1,-1)=-1, \quad \text { and } \quad \chi_{2 m}^{[P]}(1,-1)=-1, \quad m=2,3, \ldots .
$$

It can be verified that the coefficients $A_{2 m, j}^{[P]}(1,-1), j=1,2$, satisfy the five conditions in Theorem 4.7, so that these rational Gauss-Lobatto quadrature rules exist. Thus, we can again use Theorem 4.8 to compute the nodes and weights in the rational Gausstype quadrature rules.

For $\beta=\frac{4}{5}$ we find with the aid of MAPLE ${ }^{\circledR} 10^{5}$ that $J_{w}(f) \approx 0.2888276658908954$. Figure 5.1 then shows the relative error of the approximation in terms of the number of interpolation points $n$. Note that for $n=4 m+1, m=1,2, \ldots$, the rational functions in $\mathcal{L}_{n-1}=\mathcal{L}_{4 m}$ that interpolate the function $f(x)$ in the $n$th rational Gaussian nodes, have poles in the four different singularities of $f(x)$, each with the same multiplicity $m$. This may explain the oscillating behavior of the relative errors for the rational Gaussian quadrature formulas as a function of $n$, with better results for the $(4 m+1)$ th rational Gaussian quadrature formulas compared with the $(4 m+3)$ th rational Gaussian, and $(4 m+2)$ th and $(4 m+4)$ th rational Gauss-Radau quadrature formulas. Further, note that the graph for the rational Gauss-Lobatto quadrature rule (more or less) coincides with the graph for the rational Gaussian quadrature rule when shifting the latter to the right (the $(n+1)$ th rational Gauss-Lobatto and nth rational Gaussian quadrature formulas also have the same domain of validity). Due to this shift, we obtain that the rational Gauss-Lobatto quadrature rule performs a little bit better compared with the rational Gauss-Radau quadrature rule, although the latter has larger domain of validity.

Finally, to get an idea of the accuracy of the computed nodes and weights in the rational Gaussian and Gauss-Radau quadrature formulas, we also computed the nodes and weights with the aid of the MATLAB ${ }^{\circledR}$ function rcheb, as described in [20]. For each $n$, the difference in absolute value between the nodes and weights computed by means of the (modified) GEP and the nodes and weights computed by means of the MATLAB ${ }^{\circledR}$ function rcheb was less than $5.22 e-015$ and $1.32 e-013$ respectively. On the other hand, for the rational Gauss-Lobatto quadrature formulas the difference in absolute value between the fixed nodes and the nearest computed nodes was less than $2.11 e-015$, while the absolute error of the approximation of $J_{w}\left(\left|\varphi_{0}\right|^{2}\right)=1$ was less than $9.66 e-014$.

\footnotetext{
${ }^{5}$ MAPLE is a registered trademark of Waterloo Maple, Inc.
} 


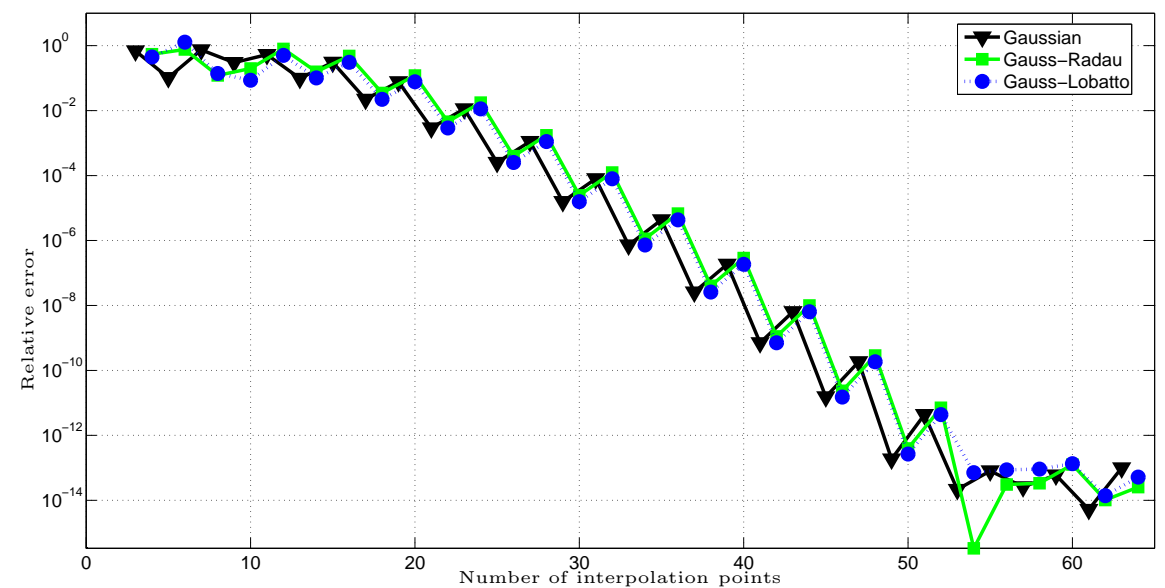

FIG. 5.1. Relative error in the rational Gauss-type quadrature formulas for the estimation of $J_{w}(f)$, where $f$ is given by (5.2).

\section{REFERENCES}

[1] A. Bultheel, P. González Vera, E. Hendriksen, and O. Njåstad. Orthogonal Rational Functions, volume 5 of Cambridge Monographs on Applied and Computational Mathematics, Cambridge University Press, Cambridge, 1999.

[2] A. Bultheel, P. González-Vera, E. Hendriksen, and O. Njåstad. "Orthogonal rational functions and quadrature on the real half line", Journal of Complexity 19(3):212-230, 2003.

[3] K. Deckers and A. Bultheel. "Orthogonal rational functions with complex poles: The Favard theorem", Journal of Mathematical Analysis and Applications 356(2):764-768, 2009.

[4] K. Deckers and A. Bultheel. "Recurrence and asymptotics for orthogonal rational functions on an interval", IMA Journal of Numerical Analysis 29(1):1-23, 2009.

[5] K. Deckers, A. Bultheel, R. Cruz-Barosso, and F. Perdomo-Pío. "Positive rational interpolatory quadrature formulas on the unit circle and the interval", Applied Numerical Mathematics, 2010. (Accepted)

[6] K. Deckers, A. Bultheel, and J. Van Deun. "A generalized eigenvalue problem for quasiorthogonal rational functions", Technical Report TW571, Department of Computer Science, K.U.Leuven, August 2010.

[7] K. Deckers, J. Van Deun, and A. Bultheel. "Computing rational Gauss-Chebyshev quadrature formulas with complex poles: The algorithm", Advances in Engineering Software 40(8):707-717, 2009.

[8] K. Deckers, J. Van Deun, and A. Bultheel. "Rational Gauss-Chebyshev quadrature formulas for complex poles outside [-1,1]", Mathematics of Computation 77(262):967-983, 2008.

[9] M. M. Džrbašian. "A survey on the theory of orthogonal systems and some open problems", in Orthogonal polynomials: Theory and practice, P. Nevai, editor, volume 294 of NATO-ASI Series C: Mathematical and Physical Sciences, pp. 135-146, Kluwer Academic Publishers, Boston, 1990.

[10] D. Fasino and L. Gemignani. "Structured eigenvalue problems for rational gauss quadrature", Numerische Mathematik 45(1-4):195-204, 2007.

[11] W. Gautschi. "Algorithm 793: GQRAT-Gauss quadrature for rational functions", ACM Transactions on Mathematical Software 25(2):213-239, 1999.

[12] J. R. Illán González. "Gaussian rational quadrature formulas for ill-scaled integrands", Journal of Computational and Applied Mathematics 233(3):745-748, 2009.

[13] K. Müller and A. Bultheel. "Translation of the Russian paper "Orthogonal systems of rational functions on the unit circle" by M.M. Džrbašian", Technical Report TW253, Department of Computer Science, K.U.Leuven, February 1997.

[14] E. A. Rovba. "Orthogonal systems of rational functions on the segment and quadratures of Gauss-type", Mathematica Balkanica (N.S.) 13(1-2):187-198, 1999. 
[15] W. Van Assche and I. Vanherwegen. "Quadrature formulas based on rational interpolation", Mathematics of Computation 61(204):765-783, 1993.

[16] J. Van Deun. "Eigenvalue problems to compute almost optimal points for rational interpolation with prescribed poles", Numerical Algorithms 45(1-4):89-99, 2007.

[17] J. Van Deun and A. Bultheel. "Orthogonal rational functions and quadrature on an interval", Journal of Computational and Applied Mathematics 153(1-2):487-495, 2003.

[18] J. Van Deun and A. Bultheel. "Orthogonal rational functions on an interval", Technical Report TW322, Department of Computer Science, K.U.Leuven, March 2001.

[19] J. Van Deun, A. Bultheel, and P. González-Vera. "On computing rational Gauss-Chebyshev quadrature formulas", Mathematics of Computation 75(253):307-326, 2006.

[20] J. Van Deun, K. Deckers, A. Bultheel, and J.A.C. Weideman. "Algorithm 882: Near best fixed pole rational interpolation with applications in spectral methods", ACM Transactions on Mathematical Software 35(2):14:1-14:21, 2008. 